\title{
CONSERVATIVE MULTIGRID METHODS FOR TERNARY CAHN-HILLIARD SYSTEMS*
}

\author{
JUNSEOK KIM ${ }^{\dagger}$, KYUNGKEUN KANG ${ }^{\ddagger}$, AND JOHN LOWENGRUB ${ }^{\S}$
}

\begin{abstract}
We develop a conservative, second order accurate fully implicit discretization of ternary (three-phase) Cahn-Hilliard (CH) systems that has an associated discrete energy functional. This is an extension of our work for two-phase systems [13]. We analyze and prove convergence of the scheme. To efficiently solve the discrete system at the implicit time-level, we use a nonlinear multigrid method. The resulting scheme is efficient, robust and there is at most a $1^{\text {st }}$ order time step constraint for stability. We demonstrate convergence of our scheme numerically and we present several simulations of phase transitions in ternary systems.
\end{abstract}

Key words. ternary Cahn-Hilliard system, nonlinear multigrid method

\section{Introduction}

Since most commercial alloys are based on at least three components, an understanding of ternary phase transitions is of great practical importance. The ternary Cahn-Hilliard $(\mathbf{C H})$ system is the prototypical continuum model of phase separation. This system was originally proposed by Morral and Cahn [15] to model threecomponent alloys. Phase separation occurs, for example, when a single phase homogeneous system composed of three components, in thermal equilibrium (e.g. at a high temperature), is rapidly cooled to a temperature $T$ below a critical temperature $T_{c}$ where the system is unstable with respect to infinitesimal concentration fluctuations. Spinodal decomposition then takes place and the system separates into spatial regions rich in some components and poor in others.

In binary mixtures, there has been much algorithm development and many simulations of the $\mathbf{C H}$ equation (e.g. see the recent papers [5], [6], [13] for references).

In multicomponent (more than two) mixtures, there have been fewer simulations. Numerical simulations of phase transitions in multicomponent have been performed by Eyre [9], Blowey et al. ([1], [2], [3], [4], and [7]), and Copetti [8]. In [9], a modified Newton method to solve the implicit finite difference system for the solution at the new time step is used. In [7], an implicit finite element method with a non-linear Gauss-Seidel type iteration is used. In [8], an explicit finite element method is used.

One of the main difficulties in solving the $\mathbf{C H}$ system is the high-order (fourth order) time step constraints for explicit methods. Thus, implicit methods are desired. However, the solution of the implicit equation can be costly when a Newton's (or Newton-like) method is coupled with a linear solver. In addition, one would like the numerical scheme to have an associated discrete energy functional consistent with that of the continuous level. The existence of such a functional provides an additional measure of stability of the scheme. The schemes given in [7] had discrete energy functionals only for restricted values of $\Delta t$. Recently, we have developed nonlinear multigrid methods [13] to solve binary $\mathbf{C H}$ systems in which the scheme has a discrete energy functional for every value of $\Delta t$. An advantage of using a nonlinear multigrid

\footnotetext{
*Received: January 19, 2004; accepted: February 8, 2004. Communicated by Weinan E.

$\dagger$ Department of Mathematics, 103 Multipurpose Science \& Technology Bldg., University of California, Irvine, CA 92697-3875, (jskim@math.uci.edu).

$\ddagger$ Department of Mathematics, University of British Columbia, 1984 Mathematics Road, Vancouver B.C., Canada V6T 1Z2, (kkang@math.ubc.ca).

$\S$ Corresponding author. Department of Mathematics, 103 Multipurpose Science \& Technology Bldg., University of California, Irvine, CA 92697-3875, (lowengrb@math.uci.edu).
} 
method is that the scheme is much more efficient than traditional iterative solvers in solving the nonlinear equations at the implicit time step.

In this paper, we build upon on our results for two phase systems [13] and develop a finite difference scheme that inherits mass conservation and energy dissipation properties from the continuous level. It is highly desirable to have a discrete energy functional because this can be used to prove that the numerical solution is uniformly bounded with respect to the time and space step sizes. From this, it follows that the scheme is stable. We prove convergence of the numerical scheme and demonstrate $2^{\text {nd }}$ order accuracy numerically. We then apply the scheme to simulate phase transitions in ternary media. In one case, we show that a two-phase microstructure in binary media can be de-stabilized by the addition of a small amount of a third component, leading to a system in which a homogeneous mixture has the lowest energy and thus the microstructure dissolves upon addition of enough of third component. In another case, we consider a ternary system in which the $3^{\text {rd }}$ component adsorbs to an interface. The third component behaves like a surfactant in that the excess energy associated with the interface decreases as more of the component accumulates at the interface. We view this work in this paper as preparatory for studies of multiphase fluid flows with 3 or more components [12].

The contents of this paper are as follows. In Section 2, the governing equations are presented. In Section 3, we derive the discrete scheme, demonstrate the existence of a discrete energy functional and prove stability and convergence of the algorithm. In Section 4, we present numerical experiments.

\section{Governing equations}

The composition of a ternary mixture (A, B, and C) can be mapped onto an equilateral triangle (the Gibbs triangle [16]) whose corners represent $100 \%$ concentration of $\mathrm{A}, \mathrm{B}$ or $\mathrm{C}$ as shown in Fig. 2.1(a). Mixtures with components lying on lines parallel to $\overline{B C}$ contain the same percentage of $\mathrm{A}$, those with lines parallel to $\overline{A C}$ have the same percentage of $\mathrm{B}$ concentration, and analogously for the $\mathrm{C}$ concentration. In Fig. 2.1(a), the mixture at the position marked 'o' contains $60 \% \mathrm{~A}, 10 \% \mathrm{~B}$, and $30 \%$ C (The total percentage must sum to $100 \%$ ).

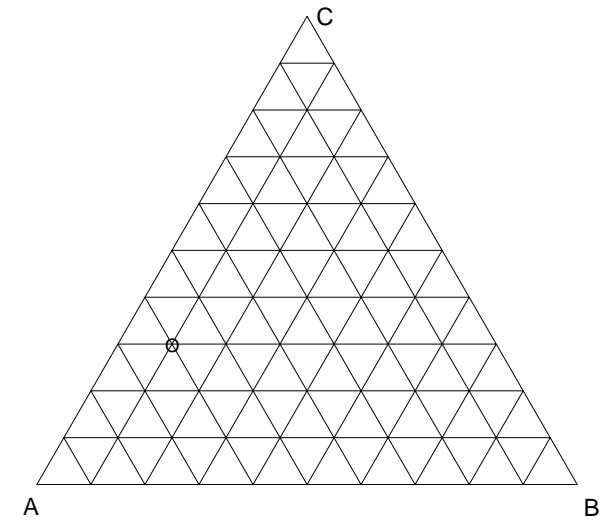

(a)

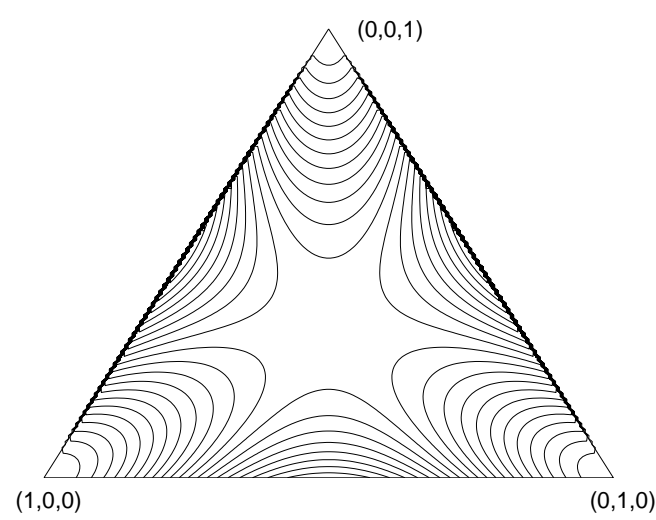

(b)

FIG. 2.1. (a) Gibbs triangle. (b) Contour plot of the free energy $F(\mathbf{c})$

Assuming that evolution is isothermal, the ternary $\mathbf{C H}$ model is as follows [15]. 
Let $\mathbf{c}=(c, d)$ be the phase variable (i.e. concentration), then

$$
\mathbf{c}_{t}(\mathbf{x}, t)=\nabla \cdot[M(\mathbf{c}) \nabla \boldsymbol{\mu}(\mathbf{x}, t)], \text { for }(\mathbf{x}, t) \in \Omega \times(0, T] \subset \mathbb{R}^{n} \times \mathbb{R}
$$

and

$$
\boldsymbol{\mu}(\mathbf{x}, t)=\mathbf{f}(\mathbf{c}(\mathbf{x}, t))-\Gamma_{\epsilon} \Delta \mathbf{c}(\mathbf{x}, t),
$$

where

$$
\mathbf{f}(\mathbf{c})=\left(f_{1}(\mathbf{c}), f_{2}(\mathbf{c})\right)=\left(\partial_{c} F(\mathbf{c}), \partial_{d} F(\mathbf{c})\right) \text { and } \quad \Gamma_{\epsilon} \equiv\left(\begin{array}{cc}
2 \epsilon^{2} & \epsilon^{2} \\
\epsilon^{2} & 2 \epsilon^{2}
\end{array}\right) .
$$

Here we denote by $\partial_{c}^{i} \partial_{d}^{j} F$ the $i$-th and $j$-th partial derivatives of $F(\mathbf{c})$ with respect to $c$ and $d$, respectively. $M(\mathbf{c})$ is the mobility, $\boldsymbol{\mu}=\left(\mu_{1}, \mu_{2}\right)$ is the generalized chemical potential, and $F(\mathbf{c})$ is the Helmholtz free energy which is nonconvex if $T<T_{c}$, to reflect the coexistence of separate phases and $\epsilon>0$ is a nondimensional measure of non-locality due to the gradient energy (Cahn number) and introduces an internal length scale (interface thickness).

Here, for simplicity, we consider a constant mobility ${ }^{1}(M \equiv 1)$ and we use the quartic free energy ${ }^{2} F(\mathbf{c})$ on the Gibbs triangle, which is defined by

$$
F(\mathbf{c})=\frac{1}{4}\left[c^{2} d^{2}+\left(c^{2}+d^{2}\right)(1-c-d)^{2}\right] .
$$

The contours of the free energy $F(\mathbf{c})$ projected onto the Gibbs triangle are shown in Fig. 2.1 (b). Note the energy minima at the vertices and the maximum at the center. Two important features of the system (2.1) and (2.2) are the conservation of the mass and the existence of a Lyapunov (energy) functional, $\mathcal{E}$, which is given by

$$
\mathcal{E}(\mathbf{c})=\int_{\Omega}\left(F(\mathbf{c})+\frac{\epsilon^{2}}{2}\left(|\nabla c|^{2}+|\nabla d|^{2}+|\nabla(1-c-d)|^{2}\right)\right) d \mathbf{x},
$$

such that

$$
\frac{d}{d t} \mathcal{E}(\mathbf{c})=-\int_{\Omega}|\nabla \boldsymbol{\mu}|^{2} d \mathbf{x}
$$

when the natural boundary conditions are applied

$$
\frac{\partial \mathbf{c}}{\partial \mathbf{n}}=\frac{\partial \boldsymbol{\mu}}{\partial \mathbf{n}}=\mathbf{0}, \quad \text { on } \partial \Omega,
$$

where $\mathbf{n}$ is the normal unit vector pointing out of $\Omega$. The initial condition is $\mathbf{c}(\mathbf{x}, 0)=$ $\mathbf{c}_{0}(\mathbf{x})$.

\section{Numerical analysis}

3.1. Discretization. We shall first discretize the ternary $\mathbf{C H}$ equation (2.1$2.2)$ in space.

Let $[a, b]$ and $[c, d]$ be partitioned by

$$
\begin{aligned}
& a=x_{\frac{1}{2}}<x_{1+\frac{1}{2}}<\cdots<x_{N_{x}-1+\frac{1}{2}}<x_{N_{x}+\frac{1}{2}}=b, \\
& c=y_{\frac{1}{2}}<y_{1+\frac{1}{2}}<\cdots<y_{N_{x}-1+\frac{1}{2}}<y_{N_{y}+\frac{1}{2}}=d,
\end{aligned}
$$

\footnotetext{
${ }^{1}$ The extension to more general $M=M(\mathbf{c})$ is straightforward.

${ }^{2}$ The extension to regular solution model free energies is straightforward [13].
} 
so that the cells

$$
I_{i j}=\left[x_{i-\frac{1}{2}}, x_{i+\frac{1}{2}}\right] \times\left[y_{j-\frac{1}{2}}, y_{j+\frac{1}{2}}\right], \quad 1 \leq i \leq N_{x}, 1 \leq j \leq N_{y}
$$

cover $\Omega=[a, b] \times[c, d]$. We denote

$$
\Delta x_{i}=x_{i+\frac{1}{2}}-x_{i-\frac{1}{2}}, \quad \Delta y_{j}=y_{j+\frac{1}{2}}-y_{j-\frac{1}{2}}
$$

and, for simplicity, we assume the above partitions are uniform in both directions, that is

$$
\Delta x_{i}=\Delta y_{j}=h \quad \text { for } \quad 1 \leq i \leq N_{x}, \quad 1 \leq j \leq N_{y}
$$

where $h=(b-a) / N_{x}=(d-c) / N_{y}$. Therefore, $x_{i+\frac{1}{2}}$ and $y_{j+\frac{1}{2}}$ can be represented as follows:

$$
x_{i+\frac{1}{2}}=a+i h, \quad y_{j+\frac{1}{2}}=c+j h .
$$

We denote by $\Omega_{h}=\left\{\left(x_{i}, y_{j}\right): 1 \leq i \leq N_{x}, \quad 1 \leq j \leq N_{y}\right\}$ the set of cell centered points $\left(x_{i}, y_{j}\right)$ where

$$
x_{i}=\frac{1}{2}\left(x_{i-\frac{1}{2}}+x_{i+\frac{1}{2}}\right), \quad y_{j}=\frac{1}{2}\left(y_{j-\frac{1}{2}}+y_{j+\frac{1}{2}}\right) .
$$

For Neumann boundary value problems, it is natural to compute numerical solutions at cell centers. Let $\mathbf{c}_{i j}$ and $\boldsymbol{\mu}_{i j}$ be approximations of $\mathbf{c}\left(x_{i}, y_{j}\right)$ and $\boldsymbol{\mu}\left(x_{i}, y_{j}\right)$. We first implement the zero Neumann boundary condition (2.4) by requiring that

$$
\begin{array}{llll}
D_{x} \mathbf{c}_{i+\frac{1}{2}, j}=0 & \text { for } i=0, & D_{x} \mathbf{c}_{i+\frac{1}{2}, j}=0 & \text { for } i=N_{x}, \\
D_{y} \mathbf{c}_{i, j+\frac{1}{2}}=0 & \text { for } j=0, & D_{y} \mathbf{c}_{i, j+\frac{1}{2}}=0 & \text { for } j=N_{y},
\end{array}
$$

where the discrete differentiation operators are

$$
D_{x} \mathbf{c}_{i+\frac{1}{2}, j}=\frac{1}{h}\left(\mathbf{c}_{i+1, j}-\mathbf{c}_{i j}\right), \quad D_{y} \mathbf{c}_{i, j+\frac{1}{2}}=\frac{1}{h}\left(\mathbf{c}_{i, j+1}-\mathbf{c}_{i j}\right) .
$$

We then define the discrete Laplacian by

$$
\Delta_{h} \mathbf{c}_{i j}=\frac{1}{h}\left(D_{x} \mathbf{c}_{i+\frac{1}{2}, j}-D_{x} \mathbf{c}_{i-\frac{1}{2}, j}\right)+\frac{1}{h}\left(D_{y} \mathbf{c}_{i, j+\frac{1}{2}}-D_{y} \mathbf{c}_{i, j-\frac{1}{2}}\right),
$$

and the discrete $L^{2}$ inner product by

$$
(\mathbf{c}, \tilde{\mathbf{c}})_{h}=h^{2} \sum_{i=1}^{N_{x}} \sum_{j=1}^{N_{y}}\left(c_{i j} \tilde{c}_{i j}+d_{i j} \tilde{d}_{i j}\right) .
$$

For a grid function $\mathbf{c}$ defined at cell centers, $D_{x} \mathbf{c}$ and $D_{y} \mathbf{c}$ are defined at cell-edges, and we use the following notation

$$
\nabla_{h} \mathbf{c}_{i j}=\left(D_{x} \mathbf{c}_{i+\frac{1}{2}, j}, D_{y} \mathbf{c}_{i, j+\frac{1}{2}}\right),
$$

to represent the discrete gradient of $\mathbf{c}$. We can define an inner product for $\nabla_{h} \mathbf{c}$ on the staggered grid by

$$
\begin{aligned}
\left(\nabla_{h} \mathbf{c}, \nabla_{h} \tilde{\mathbf{c}}\right)_{h}= & h^{2}\left(\sum_{i=0}^{N_{x}} \sum_{j=1}^{N_{y}}\left(D_{x} c_{i+\frac{1}{2}, j} D_{x} \tilde{c}_{i+\frac{1}{2}, j}+D_{x} d_{i+\frac{1}{2}, j} D_{x} \tilde{d}_{i+\frac{1}{2}, j}\right)\right. \\
& \left.+\sum_{i=1}^{N_{x}} \sum_{j=0}^{N_{y}}\left(D_{y} c_{i, j+\frac{1}{2}} D_{y} \tilde{c}_{i, j+\frac{1}{2}}+D_{y} d_{i, j+\frac{1}{2}} D_{y} \tilde{d}_{i, j+\frac{1}{2}}\right)\right) .
\end{aligned}
$$


We also define discrete norms associated with (3.2) and (3.3) as

$$
\|\mathbf{c}\|^{2}=(\mathbf{c}, \mathbf{c})_{h}, \quad|\mathbf{c}|_{1}^{2}=\left(\nabla_{h} \mathbf{c}, \nabla_{h} \mathbf{c}\right)_{h} .
$$

The time-continuous, space-discrete system that corresponds to (2.1-2.4) is

$$
\frac{d}{d t} \mathbf{c}_{i j}=\Delta_{h} \boldsymbol{\mu}_{i j}, \quad \boldsymbol{\mu}_{i j}=\mathbf{f}\left(\mathbf{c}_{i j}\right)-\Gamma_{\epsilon} \Delta_{h} \mathbf{c}_{i j}
$$

where $\mathbf{f}\left(\mathbf{c}_{i j}^{n}\right) \equiv\left(f_{1}\left(\mathbf{c}_{i j}^{n}\right), f_{2}\left(\mathbf{c}_{i j}^{n}\right)\right)$ and boundary conditions are implemented using (3.1). We discretize (3.4) in time by the scheme

$$
\begin{gathered}
\frac{\mathbf{c}_{i j}^{n+1}-\mathbf{c}_{i j}^{n}}{\Delta t}=\Delta_{h} \boldsymbol{\mu}_{i j}^{n+\frac{1}{2}}, \\
\boldsymbol{\mu}_{i j}^{n+\frac{1}{2}}=\hat{\phi}\left(\mathbf{c}_{i j}^{n}, \mathbf{c}_{i j}^{n+1}\right)-\frac{1}{2} \boldsymbol{\Gamma}_{\epsilon} \Delta_{h}\left(\mathbf{c}_{i j}^{n}+\mathbf{c}_{i j}^{n+1}\right),
\end{gathered}
$$

where $\hat{\phi}=\left(\hat{\phi}_{1}, \hat{\phi}_{2}\right)$ and $\hat{\phi}_{1}(\ldots)$ and $\hat{\phi}_{2}(\ldots)$ denote Taylor series approximations to $f_{1}$ and $f_{2}$ up to second order, respectively:

$$
\begin{aligned}
\hat{\phi}_{1}\left(\mathbf{c}^{n}, \mathbf{c}^{n+1}\right)= & f_{1}\left(\mathbf{c}^{n+1}\right)-\frac{1}{2} \partial_{c} f_{1}\left(\mathbf{c}^{n+1}\right)\left(c^{n+1}-c^{n}\right) \\
& -\frac{1}{2} \partial_{d} f_{1}\left(\mathbf{c}^{n+1}\right)\left(d^{n+1}-d^{n}\right)+\frac{1}{3 !} \partial_{c}^{2} f_{1}\left(\mathbf{c}^{n+1}\right)\left(c^{n+1}-c^{n}\right)^{2} \\
& +\frac{2}{3 !} \partial_{d} \partial_{c} f_{1}\left(\mathbf{c}^{n+1}\right)\left(c^{n+1}-c^{n}\right)\left(d^{n+1}-d^{n}\right)+\frac{1}{3 !} \partial_{d}^{2} f_{1}\left(\mathbf{c}^{n+1}\right)\left(d^{n+1}-d^{n}\right)^{2}
\end{aligned}
$$

and

$$
\begin{aligned}
\hat{\phi}_{2}\left(\mathbf{c}^{n}, \mathbf{c}^{n+1}\right)= & f_{2}\left(\mathbf{c}^{n+1}\right)-\frac{1}{2} \partial_{c} f_{2}\left(\mathbf{c}^{n+1}\right)\left(c^{n+1}-c^{n}\right) \\
& -\frac{1}{2} \partial_{d} f_{2}\left(\mathbf{c}^{n+1}\right)\left(d^{n+1}-d^{n}\right)+\frac{1}{3 !} \partial_{c}^{2} f_{2}\left(\mathbf{c}^{n+1}\right)\left(c^{n+1}-c^{n}\right)^{2} \\
& +\frac{2}{3 !} \partial_{d} \partial_{c} f_{2}\left(\mathbf{c}^{n+1}\right)\left(c^{n+1}-c^{n}\right)\left(d^{n+1}-d^{n}\right)+\frac{1}{3 !} \partial_{d}^{2} f_{2}\left(\mathbf{c}^{n+1}\right)\left(d^{n+1}-d^{n}\right)^{2} .
\end{aligned}
$$

Although these series expansions result in somewhat complicated expressions, they are easy to implement and the expansions allow us to prove that the fully discrete scheme has a non-increasing energy functional for any value of the time step $\Delta t$. In contrast, in Appendix B, we introduce an alternative (Crank-Nicholson) scheme, in which the scheme is much more straightforward. However, we are only able to prove that the Crank-Nicholson scheme has an associated non-increasing energy for restricted values of $\Delta t[11]$.

3.2. Analysis of Scheme. In this subsection, assuming that the nonlinear system at the implicit time step is solvable, we establish the mass conservation and demonstrate that the energy functional is non-increasing in time. Moreover, we demonstrate the convergence of the scheme at a fixed time. We first show the mass conservation and energy dissipation in the next Lemma.

LEMMA 3.1. If $\left\{\mathbf{c}^{n+1}, \boldsymbol{\mu}^{n+1}\right\}$ is the solution of (3.5) and (3.6) and the discrete energy functional is given by

$$
\mathcal{E}\left(\mathbf{c}^{n}\right)=\left(F\left(\mathbf{c}^{n}\right), 1\right)_{h}+\frac{\epsilon^{2}}{2}\left\|\nabla_{h} \mathbf{c}^{n}\right\|_{m}^{2}
$$


where

$$
\begin{aligned}
\left\|\nabla_{h} \mathbf{c}^{n}\right\|_{m}^{2}: & =\left|c^{n}\right|_{1}^{2}+\left|d^{n}\right|_{1}^{2}+\left|1-c^{n}-d^{n}\right|_{1}^{2} \\
& =2\left|c^{n}\right|_{1}^{2}+2\left|d^{n}\right|_{1}^{2}+2\left(\nabla_{h} c^{n}, \nabla_{h} d^{n}\right) .
\end{aligned}
$$

Then

$$
\left(\mathbf{c}^{n+1}, 1\right)_{h}=\left(\mathbf{c}^{n}, 1\right)_{h}
$$

and

$$
\mathcal{E}\left(\mathbf{c}^{n+1}\right)-\mathcal{E}\left(\mathbf{c}^{n}\right) \leq-\Delta t\left|\boldsymbol{\mu}^{n+\frac{1}{2}}\right|_{1}^{2}-\mathcal{R}_{h}\left(\mathbf{c}^{n}, \mathbf{c}^{n+1}\right)
$$

where

$$
\begin{aligned}
\mathcal{R}_{h}\left(\mathbf{c}^{n+1}, \mathbf{c}^{n}\right)= & \frac{1}{4}\left(\left(\left\|c^{n+1}-c^{n}\right\|^{2}+\left\|d^{n+1}-d^{n}\right\|^{2}\right)\left\|c^{n+1}-c^{n}+d^{n+1}-d^{n}\right\|^{2}\right. \\
& \left.\left.+\left\|c^{n+1}-c^{n}\right\|^{2}\left\|d^{n+1}-d^{n}\right\|^{2}\right)\right) .
\end{aligned}
$$

Proof. The mass conservation is straightforward by using summation by parts. Indeed,

$$
\left(\mathbf{c}^{n+1}, 1\right)_{h}=\left(\mathbf{c}^{n}+\Delta t \Delta_{h}\left(\boldsymbol{\mu}^{n}+\boldsymbol{\mu}^{n+1}\right), 1\right)_{h}=\left(\mathbf{c}^{n}, 1\right)_{h} .
$$

It remains to show the second assertion. First, multiplying $\boldsymbol{\mu}^{n+\frac{1}{2}}$ and $\mathbf{c}^{n+1}-\mathbf{c}^{n}$ to (3.5) and (3.6), we obtain the following two identities:

$$
\begin{gathered}
\left(\mathbf{c}^{n+1}-\mathbf{c}^{n}, \boldsymbol{\mu}^{n+\frac{1}{2}}\right)_{h}+\Delta t\left|\boldsymbol{\mu}^{n+\frac{1}{2}}\right|_{1}^{2}=0, \\
\left(\boldsymbol{\mu}^{n+\frac{1}{2}}, \mathbf{c}^{n+1}-\mathbf{c}^{n}\right)_{h}=\left(\hat{\phi}\left(\mathbf{c}^{n}, \mathbf{c}^{n+1}\right), \mathbf{c}^{n+1}-\mathbf{c}^{n}\right)_{h}+\frac{\epsilon^{2}}{2}\left(\left\|\nabla_{h} \mathbf{c}^{n+1}\right\|_{m}^{2}-\left\|\nabla_{h} \mathbf{c}^{n}\right\|_{m}^{2}\right) .
\end{gathered}
$$

Since the first identity (3.10) is straightforward, we only verify the second one (3.11). Indeed,

$$
\begin{aligned}
\left(\boldsymbol{\mu}^{n+\frac{1}{2}}, \mathbf{c}^{n+1}-\mathbf{c}^{n}\right)_{h} & =\left(\hat{\phi}\left(\mathbf{c}^{n}, \mathbf{c}^{n+1}\right)-\frac{1}{2} \boldsymbol{\Gamma}_{\epsilon}\left(\Delta \mathbf{c}^{n+1}+\Delta \mathbf{c}^{n}\right), \mathbf{c}^{n+1}-\mathbf{c}^{n}\right)_{h} \\
& =\left(\hat{\phi}\left(\mathbf{c}^{n}, \mathbf{c}^{n+1}\right), \mathbf{c}^{n+1}-\mathbf{c}^{n}\right)_{h}-\frac{1}{2} \boldsymbol{\Gamma}_{\epsilon}\left(\left(\Delta \mathbf{c}^{n+1}+\Delta \mathbf{c}^{n}\right), \mathbf{c}^{n+1}-\mathbf{c}^{n}\right)_{h}
\end{aligned}
$$

The second term on the right side is calculated as follows:

$$
\begin{aligned}
& \left(\boldsymbol{\Gamma}_{\epsilon}\left(\Delta \mathbf{c}^{n+1}+\Delta \mathbf{c}^{n}\right), \mathbf{c}^{n+1}-\mathbf{c}^{n}\right) \\
= & \left(\begin{array}{cc}
2 \epsilon^{2} & \epsilon^{2} \\
\epsilon^{2} & 2 \epsilon^{2}
\end{array}\right)\left(\begin{array}{c}
\Delta c^{n+1}+\Delta c^{n} \\
\Delta d^{n+1}+\Delta d^{n}
\end{array}\right) \cdot\left(\begin{array}{c}
c^{n+1}-c^{n} \\
d^{n+1}-d^{n}
\end{array}\right) \\
= & -2 \epsilon^{2}\left(\left|\mathbf{c}^{n+1}\right|_{1}^{2}-\left|\mathbf{c}^{n}\right|_{1}^{2}\right)-2 \epsilon^{2}\left(\nabla_{h} d^{n+1}, \nabla_{h} c^{n+1}\right)+2 \epsilon^{2}\left(\nabla_{h} d^{n}, \nabla_{h} c^{n}\right) \\
= & -2 \epsilon^{2}\left(\left|\mathbf{c}^{n+1}\right|_{1}^{2}+\left(\nabla_{h} d^{n+1}, \nabla_{h} c^{n+1}\right)\right)+2 \epsilon^{2}\left(\left|\mathbf{c}^{n}\right|_{1}^{2}+\left(\nabla_{h} d^{n}, \nabla_{h} c^{n}\right)\right) \\
= & -\epsilon^{2}\left\|\nabla_{h} \mathbf{c}^{n+1}\right\|_{m}^{2}+\epsilon^{2}\left\|\nabla_{h} \mathbf{c}^{n}\right\|_{m}^{2} .
\end{aligned}
$$


This completes the derivation of (3.11). Now we consider

$$
\begin{aligned}
\mathcal{E}\left(\mathbf{c}^{n+1}\right) & -\mathcal{E}\left(\mathbf{c}^{n}\right)=\left(F\left(\mathbf{c}^{n+1}\right)-F\left(\mathbf{c}^{n}\right), 1\right)_{h}+\frac{\epsilon^{2}}{2}\left(\left\|\nabla_{h} \mathbf{c}^{n+1}\right\|_{m}^{2}-\left\|\nabla_{h} \mathbf{c}^{n}\right\|_{m}^{2}\right) \\
& =\left(F\left(\mathbf{c}^{n+1}\right)-F\left(\mathbf{c}^{n}\right), 1\right)_{h}+\left(\boldsymbol{\mu}^{n+\frac{1}{2}}-\hat{\phi}\left(\mathbf{c}^{n}, \mathbf{c}^{n+1}\right), \mathbf{c}^{n+1}-\mathbf{c}^{n}\right)_{h} \\
& =\left(F\left(\mathbf{c}^{n+1}\right)-F\left(\mathbf{c}^{n}\right), 1\right)_{h}-\left(\hat{\phi}\left(\mathbf{c}^{n+1}, \mathbf{c}^{n}\right), \mathbf{c}^{n+1}-\mathbf{c}^{n}\right)_{h}-\Delta t\left|\boldsymbol{\mu}^{n+\frac{1}{2}}\right|_{1}^{2},
\end{aligned}
$$

where we used the identities (3.10) and (3.11). We abbreviate $F\left(c^{n+1}, d^{n+1}\right)=F^{n+1}$ for simplicity. Using Taylor expansions, we have

$$
\begin{aligned}
& \left(F^{n+1}-F^{n}, 1\right)_{h}-\left(\hat{\phi}\left(\mathbf{c}^{n}, \mathbf{c}^{n+1}\right), \mathbf{c}^{n+1}-\mathbf{c}^{n}\right)_{h}=-\frac{1}{4 !}\left[\left(\partial_{c}^{4} F^{n+1},\left(c^{n+1}-c^{n}\right)^{4}\right)_{h}\right. \\
& +4\left(\partial_{c}^{3} \partial_{d} F^{n+1},\left(c^{n+1}-c^{n}\right)^{3}\left(d^{n+1}-d^{n}\right)\right)_{h}+6\left(\partial_{c}^{2} \partial_{d}^{2} F^{n+1},\left(c^{n+1}-c^{n}\right)^{2}\left(d^{n+1}-d^{n}\right)^{2}\right)_{h} \\
& \left.+4\left(\partial_{c} \partial_{d}^{3} F^{n+1},\left(c^{n+1}-c^{n}\right)\left(d^{n+1}-d^{n}\right)^{3}\right)_{h}+\left(\partial_{d}^{4} F^{n+1},\left(d^{n+1}-d^{n}\right)^{4}\right)_{h}\right] \\
= & -\frac{1}{4}\left[\left(1,\left(c^{n+1}-c^{n}\right)^{4}\right)_{h}+2\left(1,\left(c^{n+1}-c^{n}\right)^{3}\left(d^{n+1}-d^{n}\right)\right)_{h}+\left(1,\left(c^{n+1}-c^{n}\right)^{4}\right)_{h}\right. \\
& \left.+2\left(1,\left(c^{n+1}-c^{n}\right)\left(d^{n+1}-d^{n}\right)^{3}\right)_{h}+3\left(1,\left(c^{n+1}-c^{n}\right)^{2}\left(d^{n+1}-d^{n}\right)^{2}\right)_{h}\right] \\
= & -\frac{1}{4}\left(\left(\left(c^{n+1}-c^{n}\right)^{2}+\left(d^{n+1}-d^{n}\right)^{2}\right)\left(c^{n+1}-c^{n}+d^{n+1}-d^{n}\right)^{2}\right. \\
& \left.+\left(c^{n+1}-c^{n}\right)^{2}\left(d^{n+1}-d^{n}\right)^{2}\right),
\end{aligned}
$$

where we used $\partial_{c}^{4} F=6, \partial_{c}^{3} \partial_{d} F=3, \partial_{c}^{2} \partial_{d}^{2} F=3, \partial_{c} \partial_{d}^{3} F=3$, and $\partial_{d}^{4} F=6$. Note that the last term is non-positive. Therefore, using the identity above, we have

$$
\mathcal{E}\left(\mathbf{c}^{n+1}\right)-\mathcal{E}\left(\mathbf{c}^{n}\right) \leq-\Delta t\left|\boldsymbol{\mu}^{n+\frac{1}{2}}\right|_{1}^{2}-\mathcal{R}_{h}\left(\mathbf{c}^{n}, \mathbf{c}^{n+1}\right) .
$$

This completes the proof of assertion (3.9).

Next we demonstrate the convergence of the scheme at a fixed time. Let $\mathbf{u}^{n}$ denote the continuous solution and $\mathbf{c}^{n}=\left(c^{n}, d^{n}\right)$ discrete solution, respectively and we denote $\mathbf{e}^{n}=\mathbf{u}^{n}-\mathbf{c}^{n}$. Here we remark that since discrete energy is bounded, it can be easily seen that a numerical solution $\mathbf{c}^{n}$ is bounded. Since this argument is straightforward, the details are omitted. Now we are ready to prove the following error estimate.

TheOrem 3.2. Suppose $\mathbf{u}$ is smooth. Then, for any $T>0$, there exists a constant $K, \Delta t_{0}$, and $h_{0}$ depending on $T, \mathbf{f}, \hat{\phi}, \epsilon$, and smoothness of $\mathbf{u}$ such that the following error estimate holds:

$$
\left\|\mathbf{e}^{n}\right\| \leq C\left(h^{2}+\Delta t^{2}\right)
$$

for $n \Delta t \leq T$ if $h \leq h_{0}$ and $\Delta t \leq \Delta t_{0}$.

Proof. Using the numerical scheme, we obtain

$$
\begin{aligned}
\partial_{t} \mathbf{e}^{m}+\boldsymbol{\Gamma}_{\epsilon} \nabla_{h}^{4} \mathbf{e}^{m+\frac{1}{2}} & =\partial_{t} \mathbf{u}^{m}+\boldsymbol{\Gamma}_{\epsilon} \nabla_{h}^{4} \mathbf{u}^{m+\frac{1}{2}}-\nabla_{h}^{2} \hat{\phi}\left(\mathbf{c}^{m+1}, \mathbf{c}^{m}\right) \\
& =\mathbf{u}_{t}\left(t_{m+\frac{1}{2}}\right)+\boldsymbol{\Gamma}_{\epsilon} \Delta^{2} \mathbf{u}\left(t_{m+\frac{1}{2}}\right)-\nabla_{h}^{2} \hat{\phi}\left(\mathbf{c}^{m+1}, \mathbf{c}^{m}\right)+\tau^{m} \\
& =\Delta \mathbf{f}\left(\mathbf{u}^{m+\frac{1}{2}}\right)-\nabla_{h}^{2} \hat{\phi}\left(\mathbf{c}^{m+1}, \mathbf{c}^{m}\right)+\tau^{m} \\
& =\nabla_{h}^{2} \mathbf{f}\left(\mathbf{u}^{m+\frac{1}{2}}\right)-\nabla_{h}^{2} \hat{\phi}\left(\mathbf{c}^{m+1}, \mathbf{c}^{m}\right)+\tau^{m} \\
& =\nabla_{h}^{2} \mathbf{f}\left(\mathbf{u}^{m+\frac{1}{2}}\right)-\nabla_{h}^{2} \mathbf{f}\left(\mathbf{c}^{m+\frac{1}{2}}\right)+\nabla_{h}^{2} \mathbf{f}\left(\mathbf{c}^{m+\frac{1}{2}}\right)-\nabla_{h}^{2} \hat{\phi}\left(\mathbf{c}^{m+1}, \mathbf{c}^{m}\right)+\tau^{m},
\end{aligned}
$$


where $\partial_{t} \mathbf{e}^{m}=\left(\mathbf{e}^{m+1}-\mathbf{e}^{m}\right) / \Delta t, \tau^{m}$ is the discretization error, and $\left\|\tau^{m}\right\| \leq C\left(h^{2}+\right.$ $\left.\Delta t^{2}\right)$. For convenience, we denote

$$
A \equiv \mathbf{f}\left(\mathbf{u}^{m+\frac{1}{2}}\right)-\mathbf{f}\left(\mathbf{c}^{m+\frac{1}{2}}\right), \quad B \equiv \mathbf{f}\left(\mathbf{c}^{m+\frac{1}{2}}\right)-\hat{\phi}\left(\mathbf{c}^{m+1}, \mathbf{c}^{m}\right) .
$$

Forming the inner product with $\mathbf{e}^{m+\frac{1}{2}}$, using summation by parts and Young's inequality, we have

$$
\begin{aligned}
\frac{1}{2} \partial_{t}\left\|\mathbf{e}^{m}\right\|^{2}+\epsilon^{2}\left\|\nabla^{2} \mathbf{e}^{m+\frac{1}{2}}\right\|^{2} \leq & \left(A, \nabla_{h}^{2} \mathbf{e}^{m+\frac{1}{2}}\right)_{h}+\left(B, \nabla_{h}^{2} \mathbf{e}^{m+\frac{1}{2}}\right)_{h} \\
& +\left\|\mathbf{e}^{m+\frac{1}{2}}\right\|^{2}+\left\|\tau^{m}\right\|^{2}
\end{aligned}
$$

where we used

$$
\epsilon^{2}\left\|\nabla^{2} \mathbf{e}^{m+\frac{1}{2}}\right\|^{2} \leq\left(\boldsymbol{\Gamma}_{\epsilon} \nabla^{2} \mathbf{e}^{m+\frac{1}{2}}, \nabla^{2} \mathbf{e}^{m+\frac{1}{2}}\right) .
$$

We first consider the first term of the right side of (3.13). Since $\left\|\mathbf{u}^{n}\right\|_{\infty}$ and $\left\|\mathbf{c}^{n}\right\|_{\infty}$ are bounded, one can easily see that $|A| \leq C\left|\mathbf{e}^{m+\frac{1}{2}}\right|$. Therefore, we obtain

$$
\left(A, \nabla_{h}^{2} \mathbf{e}^{m+\frac{1}{2}}\right) \leq C\left(\left|\mathbf{e}^{m+\frac{1}{2}}\right|,\left|\nabla_{h}^{2} \mathbf{e}^{m+\frac{1}{2}}\right|\right) \leq C\left\|\mathbf{e}^{m+\frac{1}{2}}\right\|^{2}+\frac{\epsilon^{2}}{4}\left\|\nabla_{h}^{2} \mathbf{e}^{m+\frac{1}{2}}\right\|^{2} .
$$

It remains to estimate the second term. Using a similar argument, we obtain

$$
|B| \leq C\left|\mathbf{c}^{m+1}-\mathbf{c}^{m}\right|^{2},
$$

where $C$ depends on the boundedness of the numerical solution (see Lemma A.1 in Appendix A for the details). Using the factorization and Young's inequality, we get

$$
\left(B, \nabla_{h}^{2} \mathbf{e}^{m+\frac{1}{2}}\right) \leq C\|B\|^{2}+\frac{\epsilon^{2}}{4}\left\|\nabla_{h}^{2} \mathbf{e}^{m+\frac{1}{2}}\right\|^{2} \leq C\left\|\left(\mathbf{c}^{m+1}-\mathbf{c}^{m}\right)^{2}\right\|^{2}+\frac{\epsilon^{2}}{4}\left\|\nabla_{h}^{2} \mathbf{e}^{m+\frac{1}{2}}\right\|^{2} .
$$

The next step is to estimate $\left\|\left(\mathbf{c}^{m+1}-\mathbf{c}^{m}\right)^{2}\right\|^{2}$. Adding and subtracting the continuous solution, we have

$$
\begin{aligned}
\left\|\left(\mathbf{c}^{m+1}-\mathbf{c}^{m}\right)^{2}\right\|^{2} & \leq 2\left(\left\|\left(\mathbf{c}^{m+1}-\mathbf{c}^{m}\right)^{2}-\left(\mathbf{u}^{m+1}-\mathbf{u}^{m}\right)^{2}\right\|^{2}+\left\|\left(\mathbf{u}^{m+1}-\mathbf{u}^{m}\right)^{2}\right\|^{2}\right) \\
& \leq C\left(\left\|\mathbf{e}^{m+1}-\mathbf{e}^{m}\right\|^{2}+\left\|\left(\mathbf{u}^{m+1}-\mathbf{u}^{m}\right)^{2}\right\|^{2}\right),
\end{aligned}
$$

where we again used the fact that discrete and continuous solutions are bounded. Since the continuous solution $u$ is smooth, the second term is estimated as follows:

$$
\left\|\left(\mathbf{u}^{m+1}-\mathbf{u}^{m}\right)^{2}\right\|^{2} \leq C(\Delta t)^{4}\left\|\mathbf{u}_{t}\right\|_{\infty}^{4} .
$$

Summing up all the estimates above, we obtain

$$
\left(B, \nabla_{h}^{2} \mathbf{e}^{m+\frac{1}{2}}\right) \leq C\left\|\mathbf{e}^{m+1}-\mathbf{e}^{m}\right\|^{2}+\frac{\epsilon^{2}}{4}\left\|\nabla_{h}^{2} \mathbf{e}^{m+\frac{1}{2}}\right\|^{2}+\left\|\tau^{m}\right\|^{2},
$$

and therefore, we have

$$
\begin{aligned}
\frac{1}{2} \partial_{t}\left\|\mathbf{e}^{m}\right\|^{2}+\epsilon^{2}\left\|\nabla^{2} \mathbf{e}^{m+\frac{1}{2}}\right\|^{2} \leq & \left\|\mathbf{e}^{m+\frac{1}{2}}\right\|^{2}+\frac{\epsilon^{2}}{2}\left\|\nabla_{h}^{2} \mathbf{e}^{m+\frac{1}{2}}\right\|^{2} \\
& +C\left\|\mathbf{e}^{m+1}-\mathbf{e}^{m}\right\|^{2}+\left\|\tau^{m}\right\|^{2} .
\end{aligned}
$$


Subtracting $\frac{\epsilon^{2}}{2}\left\|\nabla_{h}^{2} \mathbf{e}^{m+\frac{1}{2}}\right\|_{2}^{2}$ and multiplying 2 to both sides in (3.15), we obtain

$$
\partial_{t}\left\|\mathbf{e}^{m}\right\|^{2}+\epsilon^{2}\left\|\nabla_{h}^{2} \mathbf{e}^{m+\frac{1}{2}}\right\|^{2} \leq C\left\|\mathbf{e}^{m+\frac{1}{2}}\right\|^{2}+C\left\|\mathbf{e}^{m+1}-\mathbf{e}^{m}\right\|^{2}+\left\|\tau^{m}\right\|^{2} .
$$

Dropping $\epsilon^{2}\left\|\nabla_{h}^{2} \mathbf{e}^{m+\frac{1}{2}}\right\|^{2}$ and summing up from 0 to $n-1$, we have

$$
\begin{aligned}
\frac{\left\|\mathbf{e}^{n}\right\|^{2}}{\Delta t} & \leq \sum_{m=0}^{n-1}\left[C\left\|\mathbf{e}^{m+\frac{1}{2}}\right\|^{2}+C\left\|\mathbf{e}^{m+1}-\mathbf{e}^{m}\right\|^{2}+\left\|\tau^{m}\right\|^{2}\right] \\
& \leq \sum_{m=0}^{n-1}\left[C\left\|\mathbf{e}^{m+1}\right\|^{2}+C\left\|\mathbf{e}^{m}\right\|^{2}+\left\|\tau^{m}\right\|^{2}\right] \\
& =2 C \sum_{m=0}^{n-1}\left\|\mathbf{e}^{m}\right\|^{2}+C\left\|\mathbf{e}^{n}\right\|^{2}+n\|\tau\|^{2}
\end{aligned}
$$

where $\tau=\max _{0 \leq k \leq n-1}\left\|\tau^{k}\right\|$. Multiplying $\Delta t$ to both sides and simplifying, we obtain

$$
\begin{aligned}
(1-C \Delta t)\left\|\mathbf{e}^{n}\right\|^{2} & \leq C \Delta t \sum_{m=0}^{n-1}\left\|\mathbf{e}^{m}\right\|^{2}+(n \Delta t)\|\tau\|^{2} \\
& \leq C \Delta t \sum_{m=0}^{n-1}\left\|\mathbf{e}^{m}\right\|^{2}+T\|\tau\|^{2} \\
& \leq C \Delta t \sum_{m=0}^{n-1}\left\|\mathbf{e}^{m}\right\|^{2}+C T\left(h^{2}+\Delta t^{2}\right)
\end{aligned}
$$

where we used the fact that $n \Delta t \leq T$ and $\|\tau\| \leq C\left(h^{2}+\Delta t^{2}\right)$. Since $\Delta t$ can be chosen such that $1-C \Delta t>0$, according to the discrete version of Gronwall's inequality, we obtain $\left\|\mathbf{e}^{n}\right\| \leq C\left(h^{2}+\Delta t^{2}\right)$. This completes the proof. $\mathbf{u}$

3.3. Numerical solution. We use a nonlinear Full Approximation Storage (FAS) multigrid method to solve the nonlinear discrete system (3.5) and (3.6) at the implicit time level. The nonlinearity is treated using one step of Newton's iteration and a pointwise Gauss-Seidel relaxation scheme is used as the smoother in the multigrid method. This is a generalization of two-phase FAS Cahn-Hilliard equation solver we developed in [13]. Following a similar analysis as in [13], it can be shown that the convergence of the multigrid method can be achieved with $\Delta t \leq \Delta t_{0}$, where $\Delta t_{0}$ depends only on physical parameters and is independent of the grid size. Typically, we take $\Delta t \sim \Delta x$ to be safe. We describe the algorithm in Appendix C in detail for completeness.

\section{Numerical experiments}

4.1. Convergence test. We consider a ternary system in a one dimensional domain, $\Omega=[0,1]$. To obtain an estimate of the rate of convergence, we perform a number of simulations for a sample initial problem on a set of increasingly finer grids. The initial data is

$$
c(x)=d(x)=0.25+0.01 \cos (3 \pi x)+0.04 \cos (5 \pi x) \text { on } \Omega=[0,1] .
$$

The numerical solutions are computed on the uniform grids, $\Delta x=1 / 2^{n}$ for $n=$ $6,7,8,9$, and 10. For each case, the calculations are run to time $T=0.2$, the uniform time steps, $\Delta t=0.1 \Delta x$ and $\epsilon=0.005$, are used to establish the convergence rates. 


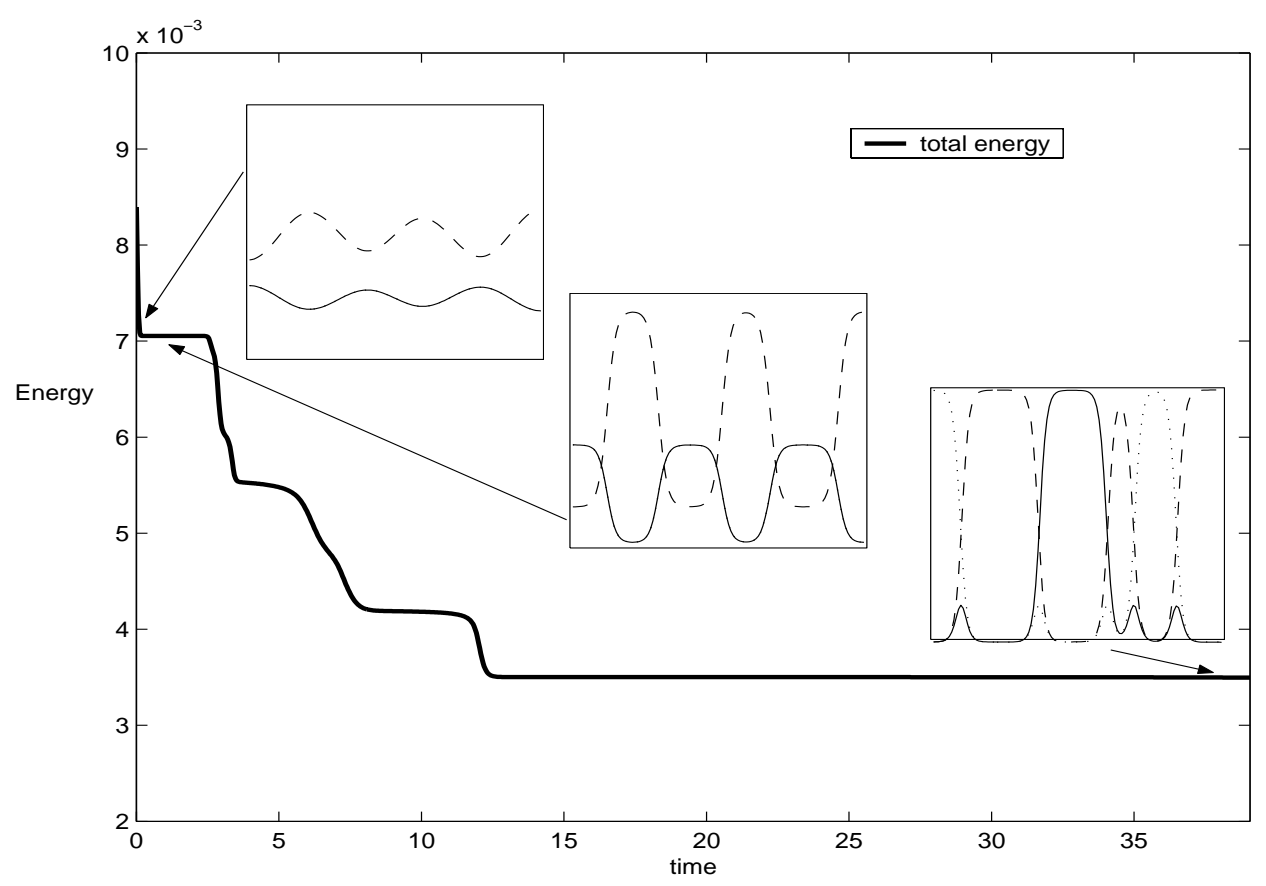

FIG. 4.1. The time dependent total energy of the numerical solutions with the initial data (4.1).

Since we use a cell centered grid, we define the error to be the discrete $l_{2}$-norm of the difference between that grid and the average of the next finer grid cells covering it:

$$
e_{h / \frac{h}{2} i} \stackrel{\text { def }}{=} c_{h i}-\left(c_{\frac{h}{2}}{ }_{2 i}+c_{\frac{h}{2}}{ }_{2 i-1}\right) / 2 .
$$

The rate of convergence is defined as the ratio of successive errors:

$$
\log _{2}\left(\left\|e_{h / \frac{h}{2}}\right\| /\left\|e_{\frac{h}{2} / \frac{h}{4}}\right\|\right)
$$

TABLE 4.1. Convergence Results - Concentration $c_{1}$.

\begin{tabular}{cccccccc}
\hline Case & $64-128$ & rate & $128-256$ & rate & $256-512$ & rate & $512-1024$ \\
\hline$l_{2}$ & $9.69 \mathrm{e}-3$ & 2.54 & $1.66 \mathrm{e}-3$ & 2.11 & $3.86 \mathrm{e}-4$ & 2.03 & $9.43 \mathrm{e}-5$ \\
\hline
\end{tabular}

The errors and rates of convergence are given in table 4.1. The results suggest that the scheme is indeed second order accurate. In Fig. 4.1, the time evolution of the energy $\mathcal{E}(\mathbf{c})$ with the same initial data (4.1) is shown accompanied with plots of concentrations (dotted line: c, solid line: d, and dashed line : 1-c-d). Note that the early stages of evolution, the curves for $\mathrm{c}$ and $\mathrm{d}$ overlap. At later times, all three phases separate. As expected from lemma 3.1, the energy is non-increasing and tends to a constant value. This is in fact a local equilibrium for Neumann boundary conditions. A global equilibrium consists of two interfaces since the components do not mix. 


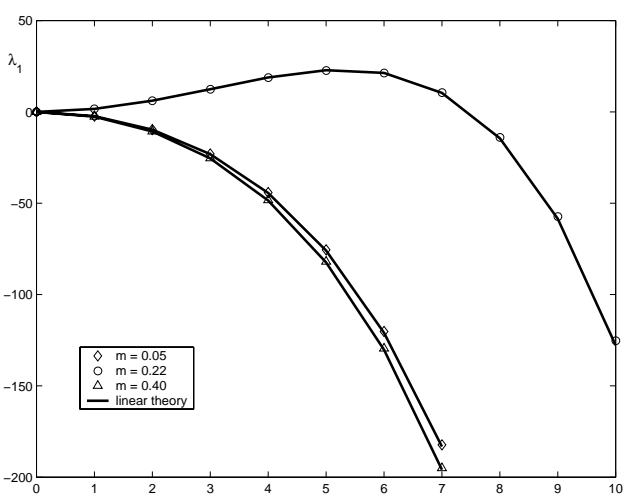

(a)

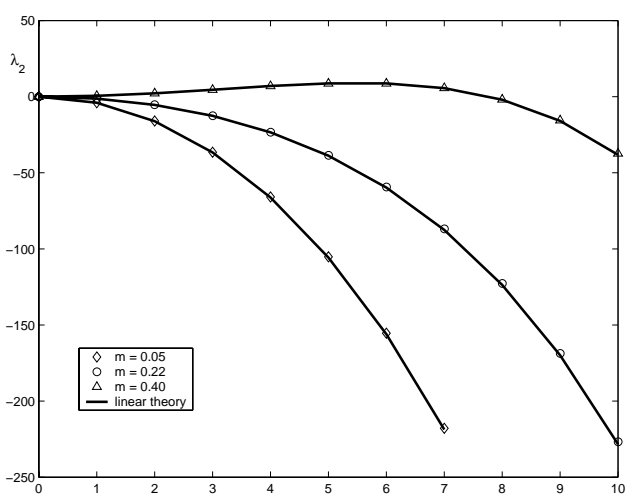

(b)

FIG. 4.2. Eigenvalues for different wave numbers $k$ with $m=0.05,0.22,0.4$ and $\epsilon=0.01$. (a): $\lambda_{1}$ and $(b): \lambda_{2}$.

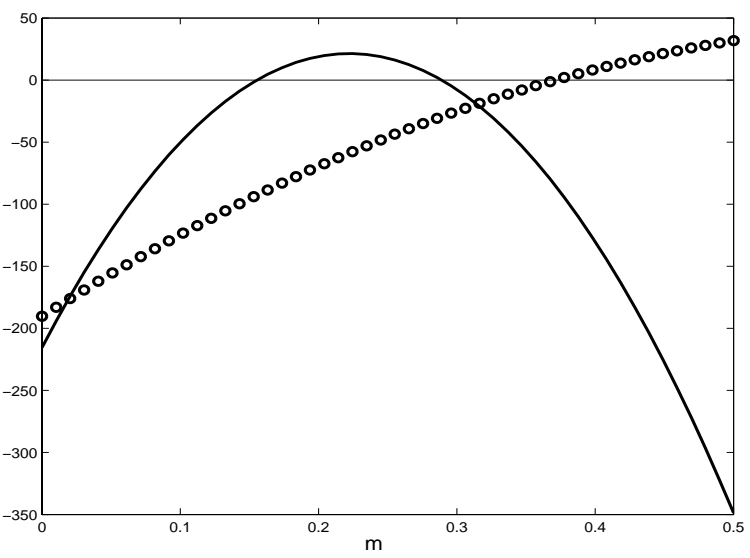

FIG. 4.3. Eigenvalues $\left(\lambda_{1}:\right.$ ' - ', $\lambda_{2}:$ ' $o$ ') with $m_{1}=m_{2}=m, k=6$, and $\epsilon=0.01$.

4.2. Linear stability analysis. Following the linear stability analysis in [7], we seek a solution of the form

$$
(c(x, t), d(x, t))=\mathbf{m}+\sum_{k=1}^{\infty} \cos (k \pi x)\left(\alpha_{k}(t), \beta_{k}(t)\right)
$$

where $\mathbf{m}=\left(m_{1}, m_{2}\right)$ and $\left|\alpha_{k}(t)\right|,\left|\beta_{k}(t)\right| \ll 1$. After linearizing $\partial_{c} F(\mathbf{c})$ and $\partial_{d} F(\mathbf{c})$ about $\mathbf{m}$, we have

$$
\begin{aligned}
& \partial_{c} F(\mathbf{c}) \approx \partial_{c} F(\mathbf{m})+\partial_{c}^{2} F(\mathbf{m})\left(c-m_{1}\right)+\partial_{c} \partial_{d} F(\mathbf{m})\left(d-m_{2}\right), \\
& \partial_{d} F(\mathbf{c}) \approx \partial_{d} F(\mathbf{m})+\partial_{c} \partial_{d} F(\mathbf{m})\left(c-m_{1}\right)+\partial_{d}^{2} F(\mathbf{m})\left(d-m_{2}\right) .
\end{aligned}
$$

Substituting these into (2.1) and (2.2) and letting $m_{1}=m_{2}=m$ for simplicity, then, up to first order, we have

$$
\begin{aligned}
c_{t} & =\left(7.5 m^{2}-4 m+0.5\right) \Delta c+\left(6 m^{2}-2 m\right) \Delta d-2 \epsilon^{2} \Delta^{2} c-\epsilon^{2} \Delta^{2} d, \\
d_{t} & =\left(6 m^{2}-2 m\right) \Delta c+\left(7.5 m^{2}-4 m+0.5\right) \Delta d-2 \epsilon^{2} \Delta^{2} d-\epsilon^{2} \Delta^{2} c .
\end{aligned}
$$


After substituting $c=m+\alpha(t) \cos (k \pi x)$ and $d=m+\beta(t) \cos (k \pi x)$ into (4.2) and (4.3), we get

$$
\left(\begin{array}{c}
\alpha_{k}(t) \\
\beta_{k}(t)
\end{array}\right)^{\prime}=\mathbf{A}\left(\begin{array}{c}
\alpha_{k}(t) \\
\beta_{k}(t)
\end{array}\right), \quad \mathbf{A}=\left(\begin{array}{cc}
a & b \\
b & a
\end{array}\right),
$$

where

$$
\begin{aligned}
& a=-(k \pi)^{2}\left(7.5 m^{2}-4 m+0.5+2(\epsilon k \pi)^{2}\right), \\
& b=-(k \pi)^{2}\left(6 m^{2}-2 m+(\epsilon k \pi)^{2}\right) .
\end{aligned}
$$

The solution to the system of $O D E \mathrm{~s}$ is given by

$$
\left(\begin{array}{c}
\alpha_{k}(t) \\
\beta_{k}(t)
\end{array}\right)=e^{\mathbf{A} t}\left(\begin{array}{c}
\alpha_{k}(0) \\
\beta_{k}(0)
\end{array}\right) \text {. }
$$

And eigenvalues of $\mathbf{A}$ are

$$
\begin{aligned}
& \lambda_{1}=-(k \pi)^{2}\left[13.5 m^{2}-6 m+0.5+3(\epsilon k \pi)^{2}\right] \\
& \lambda_{2}=-(k \pi)^{2}\left[1.5 m^{2}-2 m+0.5+(\epsilon k \pi)^{2}\right] .
\end{aligned}
$$

In Fig. 4.2(a), the theoretical growth rate $\lambda_{1}$ is compared to that obtained from the nonlinear numerical scheme with $m=0.05,0.22$, and 0.4 , initial data $c(x)=$ $d(x)=m+0.001 \cos (k \pi x), \epsilon=0.01, \Delta t=10^{-3}, h=1 / 128$ and $T=0.1$. In Fig. $4.2(\mathrm{~b})$, the theoretical growth rate $\lambda_{2}$ is compared to that obtained from the nonlinear numerical scheme with the same previous data except the initial data $c(x)=$ $m+0.001 \cos (k \pi x)$ and $d(x)=m-0.001 \cos (k \pi x)$. The numerical growth rate is defined by

$$
\tilde{\lambda}=\log \left(\frac{\max _{i}\left|c\left(x_{i}, T\right)-m\right|}{\max _{i}\left|c\left(x_{i}, 0\right)-m\right|}\right) / T .
$$

The figures show that the linear analysis (solid line) and numerical solution (symbols) are in good agreement.

To test the effect of $\mathbf{m}$, we set $k=6$ and $\epsilon=0.01$ and plotted the eigenvalues in Fig. 4.3 as a function of $m\left(m_{1}=m_{2}=m\right)$. Observe for small $m$, both eigenvalues are negative leading to decay of perturbations. The maximum growth rate for $\lambda_{1}$ occurs when $m \approx 0.2$ and for $\lambda_{2}$ occurs for $m>0.5$.

We performed three experiments with initial data taking $m=0.22,0.4$, and 0.05. We chose $\Delta x=1 / 128$ and $\Delta t=0.001$. The initial conditions were random perturbations with amplitude 0.05 of the uniform state $\mathbf{m}$.

In the first experiment (Fig. 4.4 (a)), where $m=0.22\left(\lambda_{1}>0\right.$ and $\left.\lambda_{2}<0\right)$, initially the third phase $1-c-d$ dominates. At early times, the evolution tends toward the development of a 5-mode dominated two-phase structure with $c \approx d$, which is consistent with linear theory (see Fig. $4.4(\mathrm{a}), \mathrm{t}=0.5)$. However, as the evolution proceeds, competition among the three phases leads to the development of a fully three-phase microstructure at $t=10.0$. Note the tendency of one of the components to accumulate at interfaces (see also [10]).

In the second experiment (Fig. $4.4(\mathrm{~b})), m=0.4\left(\lambda_{1}<0\right.$ and $\left.\lambda_{2}>0\right)$, the evolution again proceeds much like that of a binary system where the $c$ and $d$ phases separate creating a 6 -mode dominated microstructure while the third $(1-c-d)$ phase 

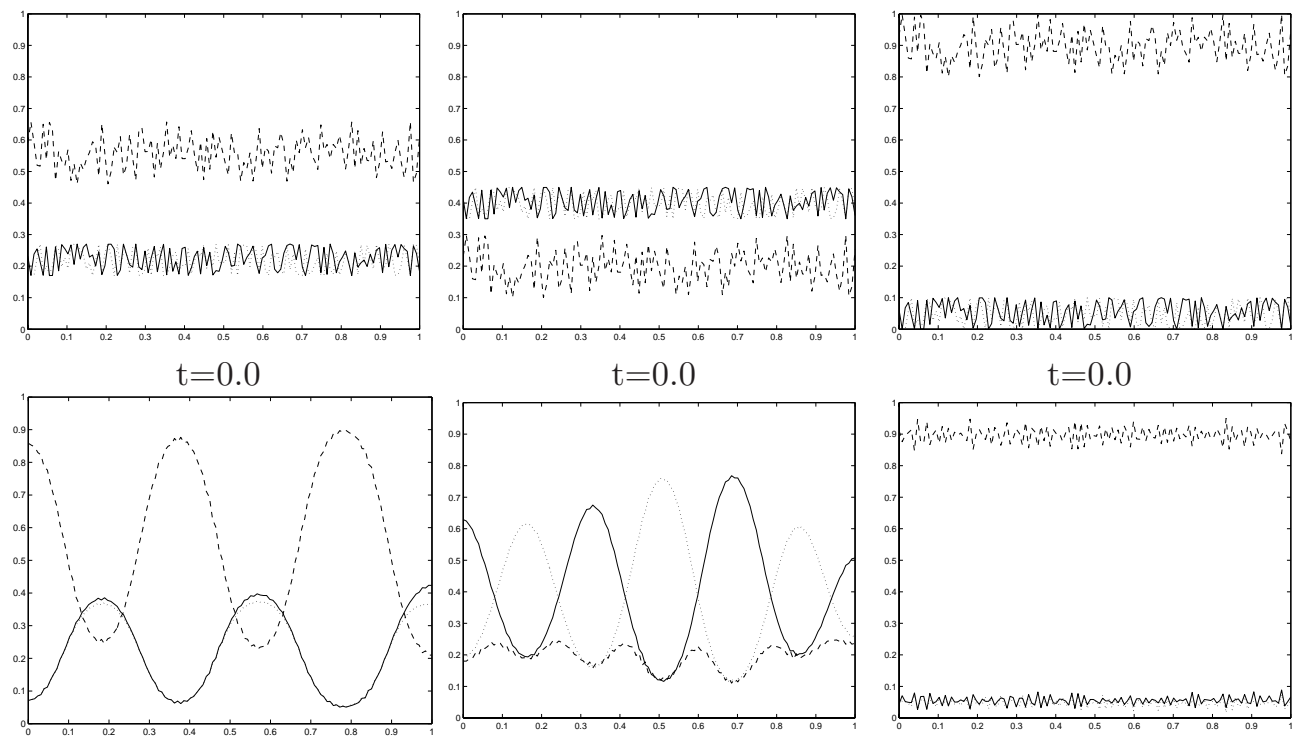

$\mathrm{t}=0.0$

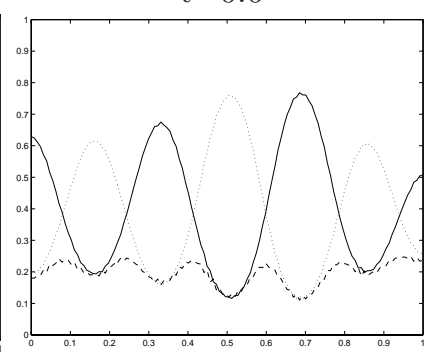

$\mathrm{t}=0.0$

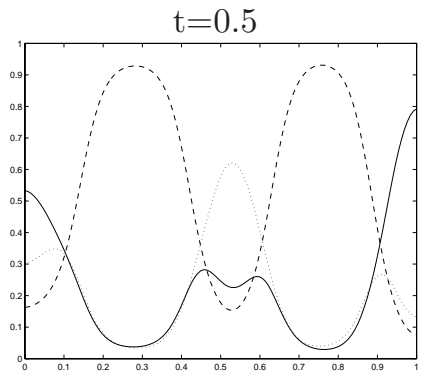

$\mathrm{t}=2.75$
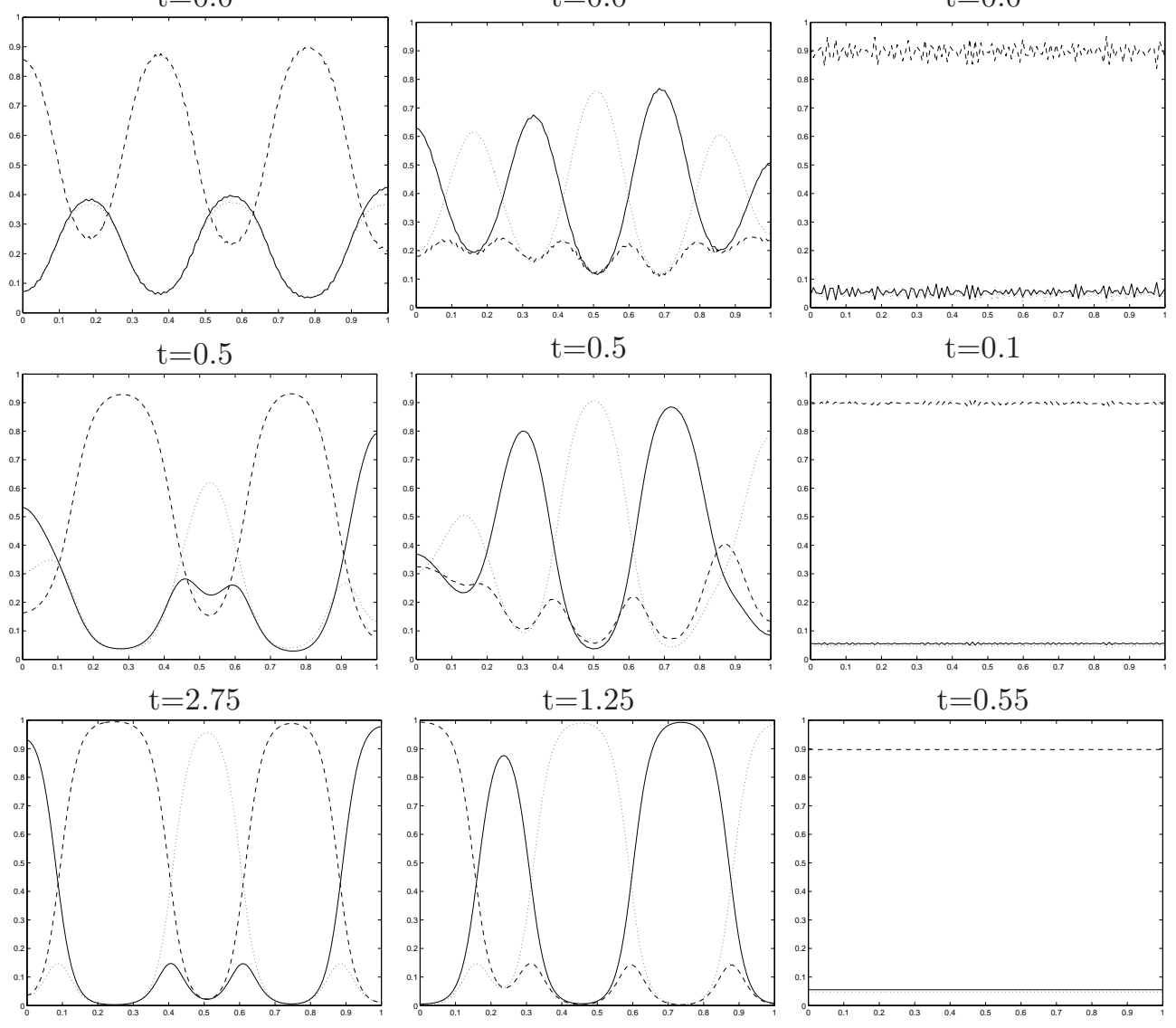

$\mathrm{t}=10.0$

(a)

$\mathrm{t}=1.25$

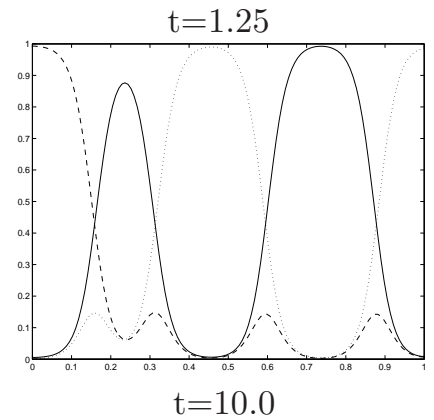

(b)

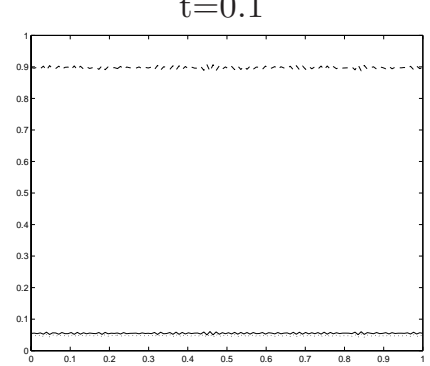

$\mathrm{t}=0.55$

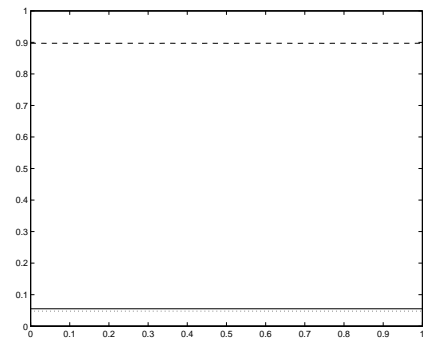

$\mathrm{t}=2.0$

(c)

FIG. 4.4. (a) $\left(m_{1}, m_{2}, m_{3}\right)=(0.22,0.22,0.56)$, (b) $\left(m_{1}, m_{2}, m_{3}\right)=(0.4,0.4,0.2)$, and (c) $\left(m_{1}, m_{2}, m_{3}\right)=(0.05,0.05,0.9)$. Solid, dotted, and dashed lines are $c, d$, and $1-c-d$, respectively.

remains nearly constant (see Fig. $4.4(\mathrm{~b}), \mathrm{t}=0.5)$. At later times $(\mathrm{t}=10.0)$, the three phases fully separate with the third phase existing at the $c$ and $d$ interfaces and in the region $0.0<x<0.15$.

In the third experiment ((Fig. $4.4(\mathrm{c}))$, where $m=0.05\left(\lambda_{1}<0\right.$ and $\left.\lambda_{2}<0\right)$, the initial perturbation is not large enough to stimulate domain growth. Instead the perturbation is damped and the evolution tends toward a homogeneous mixture (see 
Fig. $4.4(\mathrm{c}), \mathrm{t}=2.0)$.
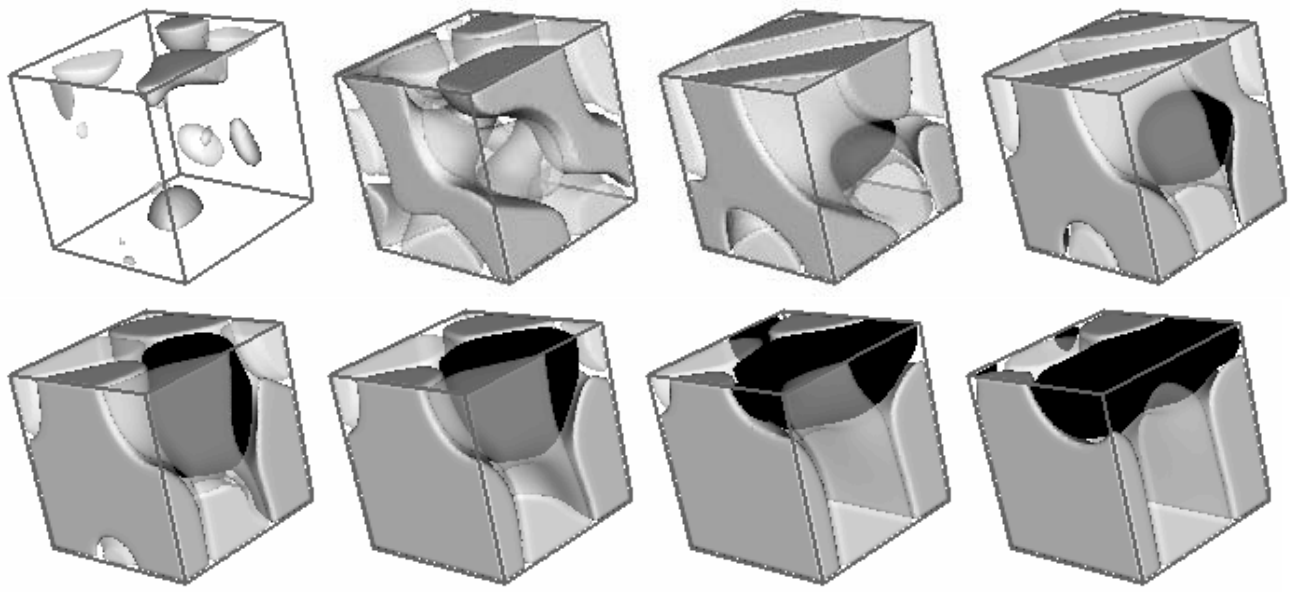

FIG. 4.5. Time evolution of half isosurface of each component is shown (transparent: c, light gray: $d$, and dark gray: $1-c-d$ ). The nondimensional times are $t=1,2,4,7,9,10$, 13, and 16 (from left to right and top to bottom order).

Since our algorithm extends straightforwardly to $3 \mathrm{D}$, we also performed three dimensional experiment with initial data taking $m=0.4$ on box size $[0,1] \times[0,1] \times$ $[0,1]$. We chose a mesh size $64 \times 64 \times 64$ and $\Delta t=0.001$. The initial conditions were random perturbations with amplitude 0.1 of the uniform state $\mathbf{m}$. Fig. 4.5 shows time evolution of the 0.5 isosurface of each component (transparent: $c$, light gray: $d$, and dark gray: $1-c-d$ ). The nondimensional times are $\mathrm{t}=1,2,4,7,9,10,13$, and 16 (from left to right and top to bottom order). As in the second case of the 1D experiments, initially a binary system (transparent and light gray phases) forms (up to time 2 in the Fig. 4.5). And then later times (from $t=4$ ), the third phase emerges.

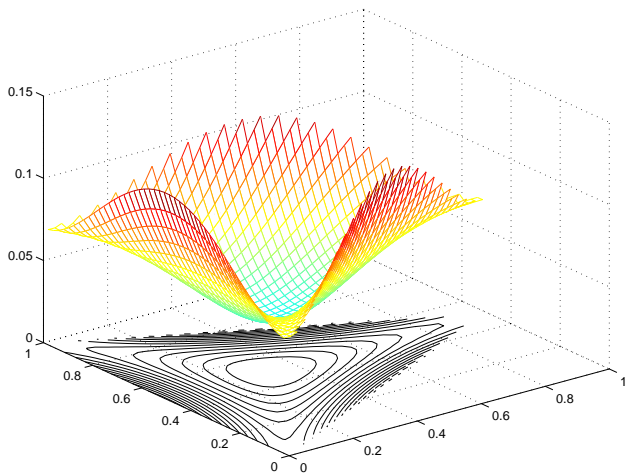

(a)

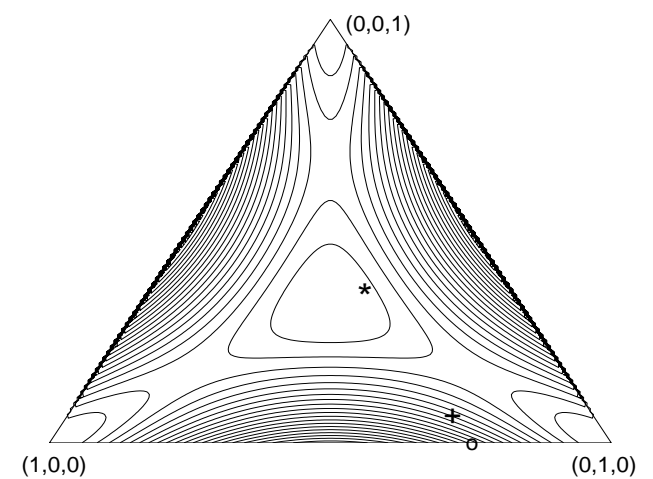

(b)

FIG. 4.6. (a) Surface and contour plots of the free energy $F(\mathbf{c})$. (b) Initial concentration, $\left(c_{1}, c_{2}, c_{3}\right)=(0.25,0.75,0)$, ०, case $1(\star)$, and case $2(+)$. 
4.3. Phase transition. Next, we study a phase transition by adding a third component to a phase-separated binary system that then results in the dissolution of the separate phases. The free energy $F(\mathbf{c})$ of the system is defined as follows:

$$
F(c, d)=\frac{1}{4}\left[c^{2} d^{2}+\left(c^{2}+d^{2}\right)(1-c-d)^{2}-c d(1-c-d)\right] .
$$

Figs. 4.6(a) and 4.6(b) show the surface and contour plots of free energy $F(c, d)$ from Eq. (4.6), respectively. When the third component is absent $(c+d=1)$, the free energy is double-welled with minima at $c=0$ and $d=0$. Thus, the binary system tends to phase-separate. When the third component is present, there is a global minimum in the center of the Gibbs triangle. Thus, the phases can mix uniformly when enough of the third component is added.

We consider an initial configuration given by

$$
\begin{aligned}
& c(x, y, 0)=0.25+0.3(0.5-\operatorname{rand}(x, y)), \\
& d(x, y, 0)=1-c(x, y, 0),
\end{aligned}
$$

where 0.25 lies in the spinodal region $\left(\frac{\partial^{2} F}{\partial c^{2}} \leq 0\right)$ for the binary system and $\operatorname{rand}(x, y)$ is a random number between 0 and 1 . The numerical parameters are $\epsilon=0.005$, $h=1 / 128, \Delta t=0.1 h$ with $N_{x}=N_{y}=128$. The initial average concentration ('o') is indicated on the Gibbs triangle in Fig. 4.6 (b). During the evolution, spinodal decomposition first occurs and then the phases separate.

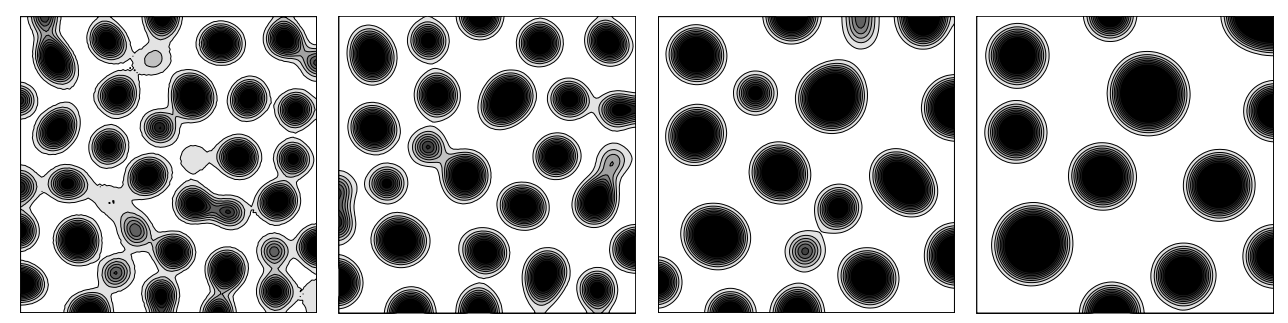

FIG. 4.7. Phase separation of binary mixture at time $t=0.12,0.20,0.66$, and 1.56 (left to right). The concentration fields are shown with filled contours at from $c=0.1$ to $c=0.9$ increased by 0.1 .

Fig. 4.7 shows the concentration $c$ at different times during the evolution. By time $t=1.56$, the binary microstructure is created. At this point the evolution is stopped and we add some of the third component as follows.

First, we replace the half of the component $d$ (in the exterior of the circular c-phase domains) with the third component. The average concentration $\left.\left({ }^{(*}\right)\right)$ is located on the Gibbs triangle in Fig. 4.6 (b). Fig. 4.8 shows the time evolution of each component during the succeeding evolution. Observe that the microstructure dissolves. Fig. 4.9 shows the evolution of the total and interface energy throughout the whole process (case 1). Note that the time scale for dissolution is much faster than that for phase separation.

In the second example (case 2), we replace $\frac{1}{10}$ of component $d$ by the third component at $t=1.56$. The average concentration (' + ') is located on the Gibbs triangle in Fig. 4.6 (b). Fig. 4.10 shows the time evolution of each component during the succeeding evolution. Observe that while the microstructure dissolves somewhat, complete dissolution does not occur. This can also be seen in Fig. 4.9 where it is 
demonstrated that the interface energy for this case remains non-zero (unlike case 1). The reason the microstructure does not completely dissolve in case 2 is that not enough of the third component was added.

4.4. Surfactant. In this section, we provide an example of microphase separation in which one of the components accumulates at an interface separating two immiscible components. The idea here is to model the effects of a surfactant. The free energy we consider here is

$$
\begin{aligned}
& \mathcal{E}(\mathbf{c})=\int_{\Omega}\left(F(\mathbf{c})+\frac{\epsilon^{2}}{2}|\nabla c|^{2}\right) d \mathbf{x} \\
& F(\mathbf{c})=\frac{1}{4} c^{2}(1-c)^{2}+s d^{2} h(c)+s\left(d-\frac{t o t}{2}\right)^{2} \\
& h(c)=1.1-0.5 \tanh \frac{c-0.2}{\epsilon}-0.5 \tanh \frac{0.8-c}{\epsilon}
\end{aligned}
$$

where $c$ represents the concentration of one of the immiscible components and $d$ the concentration of surfactant. Each of the terms in (4.9) is understood as follows. The first promotes phase-separation of the immiscible components, which are denoted by $c=0$ and $c=1$. The second term promotes the adsorption of $d$ to the interface. The third term models the miscibility of the surfactant in the immiscible components.
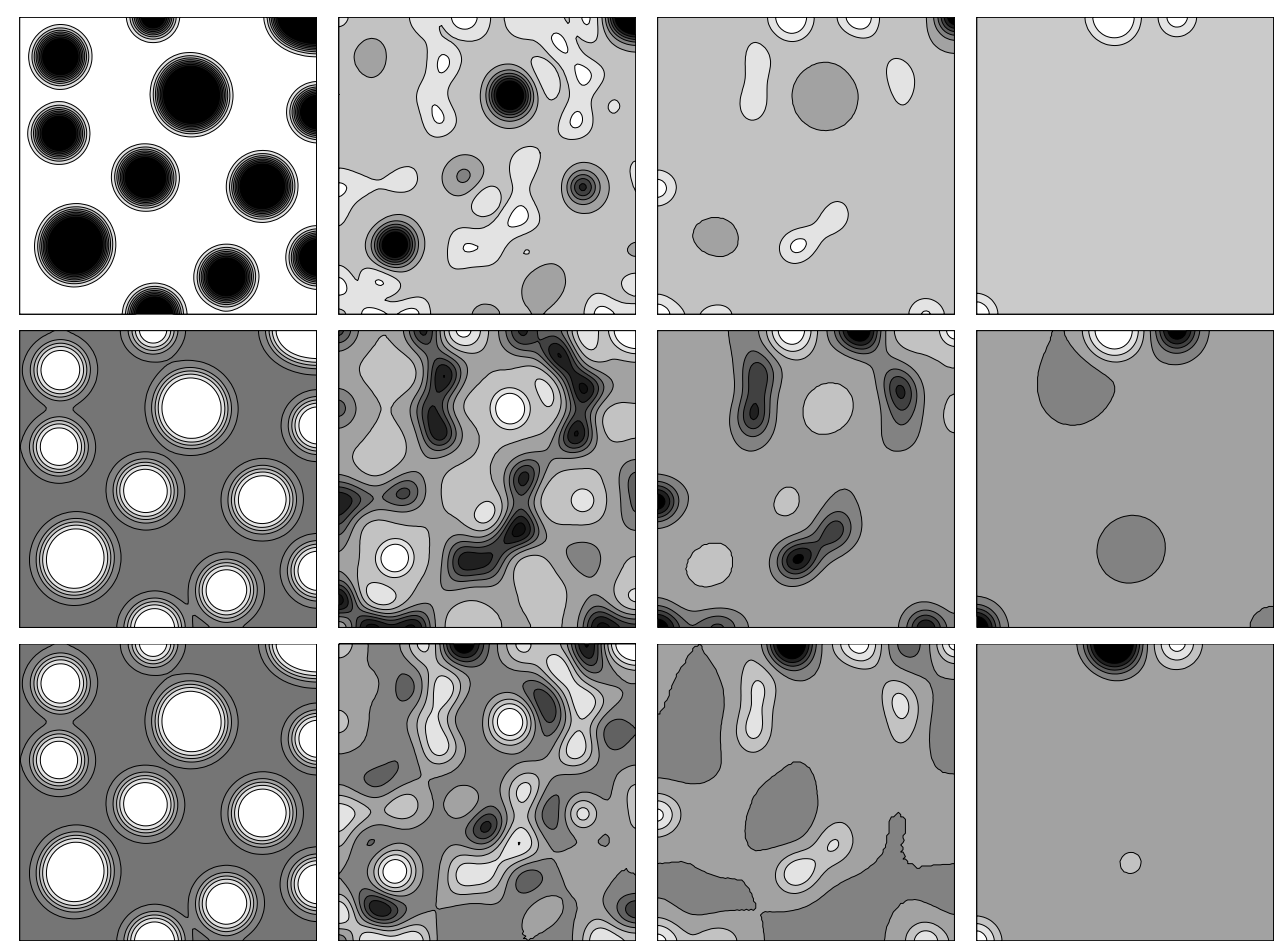

FIG. 4.8. After time $t=1.56$, concentrations are I (25\%), II (37.5\%), and III (37.5\%) Times are $t=1.56,1.60,1.68$, and 1.95 (left to right). Top: $c$; middle: d; bottom: 1-c-d. The concentration fields are shown with filled contours at from $c=0.1$ to $c=0.9$ increased by 0.1 . 


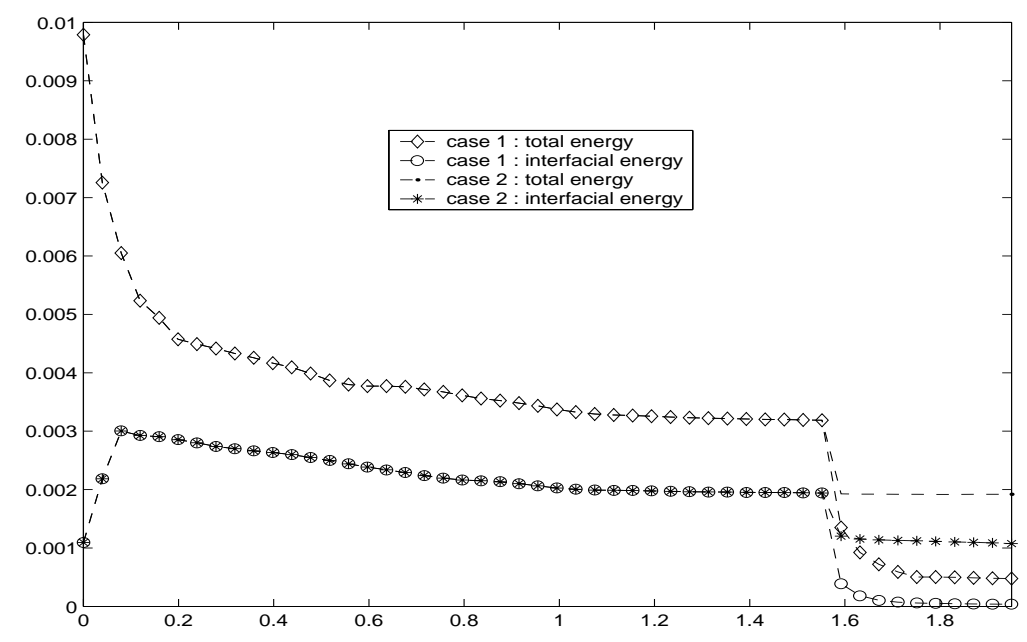

FIG. 4.9. Total energy and interfacial energy for evolution in Figs. 4.8 and 4.10
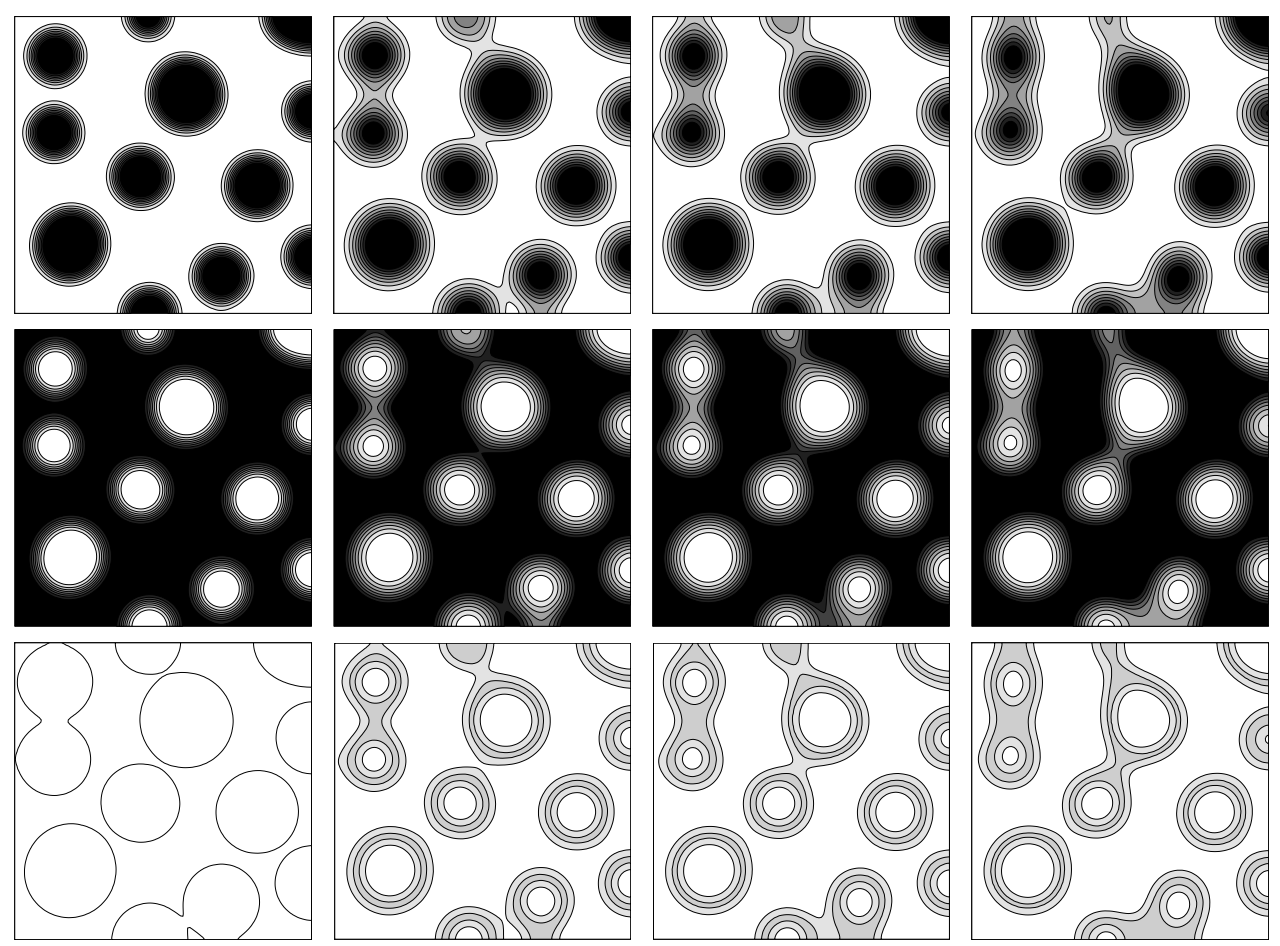

FIG. 4.10. After time $t=1.56$, concentrations are I (25\%), II (67.5\%), and III (7.5\%). Times are $t=1.56,1.68,1.80$, and 1.95 (left to right). Top: c; middle: d; bottom: 1 -c-d. The concentration fields are shown with filled contours at from $c=0.1$ to $c=0.9$ increased by 0.1 . 


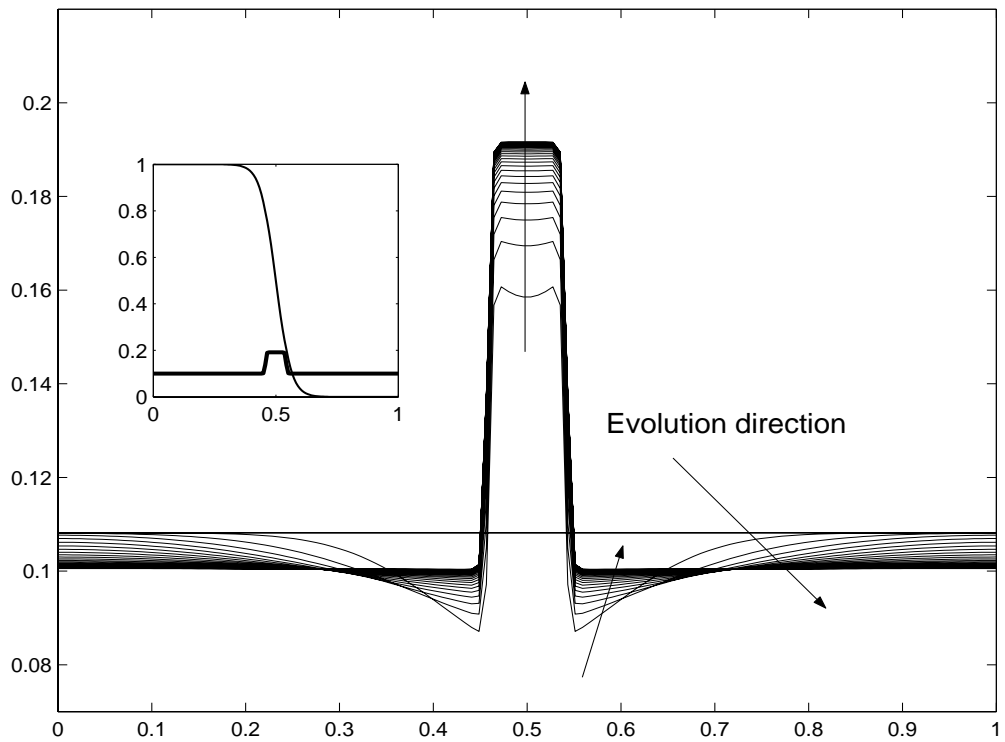

FIG. 4.11. Evolution of surfactant concentration with average concentration $d_{\text {ave }}=0.11$. The inset is an equilibrium state of interface and surfactant.

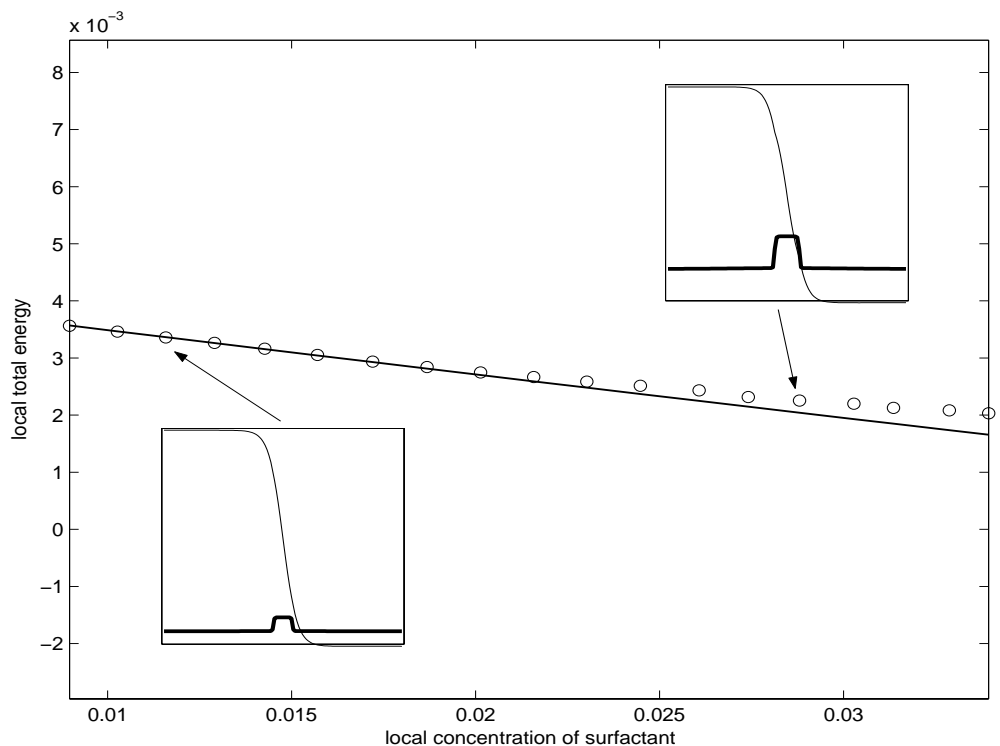

Fig. 4.12. Local total energy

Note that here since we want $d$ to accumulate at the interface, we drop the condition that the third component is given by $1-c-d$. Further, in (4.9), $s$ is a scalar factor. 
We consider an initial configuration given by

$$
\begin{aligned}
& c(x, 0)=0.5\left[1-\tanh \left(\frac{x-0.5}{2 \sqrt{2} \epsilon}\right)\right], \\
& d(x, 0)=d_{\text {ave }},
\end{aligned}
$$

where $d_{\text {ave }}$ is constant and varies from 0.058 to 0.21 in each case. The parameters are $\epsilon=0.02, s=0.1$, tot $=1, h=1 / 128, \Delta t=0.1 h$, and $N_{x}=N_{y}=128$. In Fig. 4.11, the evolution of the surfactant concentration with average concentration $d_{\text {ave }}=0.11$ is shown. Evolution directions are indicated by arrows. Observe that the surfactant rapidly absorbs to the interface and the overshoots that occur at early times flatten out.

Fig. 4.12 shows the numerical result of local total energy (symbols), which is the numerical evaluation of $\mathcal{E}(\mathbf{c})$ within interface area $(0.1 \leq c \leq 0.9)$ as a function of the surfactant concentration $\int_{0.1 \leq c \leq 0.9} d$. The solid line is the curve, $-0.01572+0.08(d-$ tot $/ 2)^{2}$. The surface tension is $0.00428-0.08 d^{2}$ [14]. The inscribed figures correspond to equilibrium states.

\section{Conclusion}

In this paper, we have developed and proved the convergence of a $2^{\text {nd }}$ order accurate finite difference numerical scheme for ternary $\mathbf{C H}$ systems. This is a natural extension of our previous work [13] on binary mixtures. The scheme has a discrete energy functional. We have used a FAS nonlinear multigrid method to solve the discrete system accurately and efficiently. We applied the scheme to simulate phase transitions in ternary media. We showed that a two-phase microstructure in binary media can be de-stabilized by the addition of a small amount of a third component, leading to a system in which a homogeneous mixture has the lowest energy and thus the dissolution of the microstructure. We also considered a ternary system in which the $3^{\text {rd }}$ component adsorbs to an interface, resulting in decreases of the excess energy associated with the interface as more of the component accumulates at the interface.

We view the work presented here as preparatory for a study of 3-component liquids. In a companion paper [12], we will couple the ternary $\mathbf{C H}$ model to the equations of fluid flow to simulate the dynamics of flows consisting 3 components.

Appendix A. Verification of (3.14). In this appendix, we verify (3.14) in Theorem 3.2. We recall $F(\mathbf{c})$, which is given by

$$
F(c, d)=\frac{1}{4}\left[c^{2} d^{2}+\left(c^{2}+d^{2}\right)(1-c-d)^{2}\right] .
$$

Then, we have

Lemma A.1.

$$
\left|\mathbf{f}\left(\mathbf{c}^{m+\frac{1}{2}}\right)-\hat{\phi}\left(\mathbf{c}^{m+1}, \mathbf{c}^{m}\right)\right| \leq C\left|\mathbf{c}^{m+1}-\mathbf{c}^{m}\right|^{2},
$$

where $C$ depends on the uniform boundedness of numerical solution $\mathbf{c}^{k}$ for all $k$.

Proof. We denote $\partial_{c}^{i} \partial_{d}^{j} F\left(\mathbf{c}^{m+1}\right)=F_{c^{i} d^{j}}^{m+1}$ for convenience of notation. We first 
expand $\mathbf{f}\left(\mathbf{c}^{m+\frac{1}{2}}\right)$ at $\mathbf{c}^{m+1}$. After simple calculations, we have

$$
\begin{aligned}
f_{1}\left(\frac{\mathbf{c}^{m+1}+\mathbf{c}^{m}}{2}\right)= & F_{c}^{m+1}+F_{c^{2}}^{m+1}\left(\frac{c^{m}-c^{m+1}}{2}\right)+F_{c d}^{m+1}\left(\frac{d^{m}-d^{m+1}}{2}\right) \\
& +\frac{1}{2} F_{c^{3}}^{m+1}\left(\frac{c^{m}-c^{m+1}}{2}\right)^{2}+F_{d c^{2}}^{m+1}\left(\frac{c^{m}-c^{m+1}}{2}\right)\left(\frac{d^{m}-d^{m+1}}{2}\right) \\
& +\frac{1}{2} F_{d^{2} c}^{m+1}\left(\frac{d^{m}-d^{m+1}}{2}\right)^{2}+\frac{1}{3 !} F_{c^{4}}^{m+1}\left(\frac{c^{m}-c^{m+1}}{2}\right)^{3} \\
& +\frac{1}{2} F_{d c^{3}}^{m+1}\left(\frac{c^{m}-c^{m+1}}{2}\right)^{2}\left(\frac{d^{m}-d^{m+1}}{2}\right) \\
& +\frac{1}{2} F_{d^{2} c^{2}}^{m+1}\left(\frac{c^{m}-c^{m+1}}{2}\right)\left(\frac{d^{m}-d^{m+1}}{2}\right)^{2}+\frac{1}{3 !} F_{d^{3} c}^{m+1}\left(\frac{d^{m}-d^{m+1}}{2}\right)^{3},
\end{aligned}
$$

and

$$
\begin{aligned}
f_{2}\left(\frac{\mathbf{c}^{m+1}+\mathbf{c}^{m}}{2}\right)= & F_{d}^{m+1}+F_{c d}^{m+1}\left(\frac{c^{m}-c^{m+1}}{2}\right)+F_{d^{2}}^{m+1}\left(\frac{d^{m}-d^{m+1}}{2}\right) \\
& +\frac{1}{2} F_{c^{2} d}^{m+1}\left(\frac{c^{m}-c^{m+1}}{2}\right)^{2}+F_{c d^{2}}^{m+1}\left(\frac{c^{m}-c^{m+1}}{2}\right)\left(\frac{d^{m}-d^{m+1}}{2}\right) \\
& +\frac{1}{2} F_{d^{3}}^{m+1}\left(\frac{d^{m}-d^{m+1}}{2}\right)^{2}+\frac{1}{3 !} F_{c^{3} d}^{m+1}\left(\frac{c^{m}-c^{m+1}}{2}\right)^{3} \\
& +\frac{1}{2} F_{c^{2} d^{2}}^{m+1}\left(\frac{c^{m}-c^{m+1}}{2}\right)^{2}\left(\frac{d^{m}-d^{m+1}}{2}\right) \\
& +\frac{1}{2} F_{c d^{3}}^{m+1}\left(\frac{c^{m}-c^{m+1}}{2}\right)\left(\frac{d^{m}-d^{m+1}}{2}\right)^{2}+\frac{1}{3 !} F_{d^{4}}^{m+1}\left(\frac{d^{m}-d^{m+1}}{2}\right)^{3} .
\end{aligned}
$$

Recalling the expression of $\hat{\phi}$, we obtain

$$
\begin{aligned}
I:= & f_{1}\left(\frac{\mathbf{c}^{m+1}+\mathbf{c}^{m}}{2}\right)-\hat{\phi}\left(\mathbf{c}^{m}, \mathbf{c}^{m+1}\right)=-\frac{1}{24} F_{c^{3}}^{m+1}\left(c^{m+1}-c^{m}\right)^{2} \\
& -\frac{1}{12} F_{d c^{2}}^{m+1}\left(c^{m+1}-c^{m}\right)\left(d^{m+1}-d^{m}\right)-\frac{1}{24} F_{d^{2} c}^{m+1}\left(d^{m+1}-d^{m}\right)^{2} \\
& -\frac{1}{48} F_{c^{4}}^{m+1}\left(c^{m+1}-c^{m}\right)^{3}-\frac{1}{16} F_{d c^{3}}^{m+1}\left(c^{m+1}-c^{m}\right)^{2}\left(d^{m+1}-d^{m}\right) \\
& -\frac{1}{16} F_{d^{2} c^{2}}^{m+1}\left(c^{m+1}-c^{m}\right)\left(d^{m+1}-d^{m}\right)^{2}-\frac{1}{48} F_{d^{3} c}^{m+1}\left(d^{m+1}-d^{m}\right)^{3} \\
= & -\frac{1}{24}\left(6 c^{m+1}+3 d^{m+1}-3\right)\left(c^{m+1}-c^{m}\right)^{2}-\frac{1}{16}\left(d^{m+1}-d^{m}\right)^{3} \\
& -\frac{1}{12}\left(3 d^{m+1}+3 c^{m+1}-1\right)\left(c^{m+1}-c^{m}\right)\left(d^{m+1}-d^{m}\right) \\
& -\frac{1}{24}\left(3 c^{m+1}+3 d^{m+1}-1\right)\left(d^{m+1}-d^{m}\right)^{2}-\frac{1}{8}\left(c^{m+1}-c^{m}\right)^{3} \\
& -\frac{3}{16}\left(c^{m+1}-c^{m}\right)^{2}\left(d^{m+1}-d^{m}\right)-\frac{3}{16}\left(c^{m+1}-c^{m}\right)\left(d^{m+1}-d^{m}\right)^{2} .
\end{aligned}
$$

Since each term is at least second order, using Young's inequality, i.e. $2 a b \leq a^{2}+b^{2}$ for $a, b \in \mathbb{R}$, we get

$$
|I| \leq C\left(\left(c^{m+1}-c^{m}\right)^{2}+\left(d^{m+1}-d^{m}\right)^{2}\right)
$$


where we used that $\mathbf{c}^{m}, \mathbf{c}^{m+1}$ are bounded. In a similar manner, we obtain

$$
\begin{aligned}
I I:= & f_{2}\left(\frac{\mathbf{c}^{m+1}+\mathbf{c}^{m}}{2}\right)-\hat{\phi}_{2}\left(\mathbf{c}^{m}, \mathbf{c}^{m+1}\right)=-\frac{1}{24} F_{c^{2} d}^{m+1}\left(c^{m+1}-c^{m}\right)^{2} \\
& -\frac{1}{12} F_{c d^{2}}^{m+1}\left(c^{m+1}-c^{m}\right)\left(d^{m+1}-d^{m}\right)-\frac{1}{24} F_{d^{3}}^{m+1}\left(d^{m+1}-d^{m}\right)^{2} \\
& -\frac{1}{48} F_{c^{3} d}^{m+1}\left(c^{m+1}-c^{m}\right)^{3}-\frac{1}{16} F_{c^{2} d^{2}}^{m+1}\left(c^{m+1}-c^{m}\right)^{2}\left(d^{m+1}-d^{m}\right) \\
& -\frac{1}{16} F_{c d^{3}}^{m+1}\left(c^{m+1}-c^{m}\right)\left(d^{m+1}-d^{m}\right)^{2}-\frac{1}{48} F_{d^{4}}^{m+1}\left(d^{m+1}-d^{m}\right)^{3} \\
= & -\frac{1}{24}\left(3 d^{m+1}+3 c^{m+1}-1\right)\left(c^{m+1}-c^{m}\right)^{2}-\frac{1}{8}\left(d^{m+1}-d^{m}\right)^{3} \\
& -\frac{1}{12}\left(3 c^{m+1}+3 d^{m+1}-1\right)\left(c^{m+1}-c^{m}\right)\left(d^{m+1}-d^{m}\right) \\
& -\frac{1}{24}\left(6 d^{m+1}+3 c^{m+1}-3\right)\left(d^{m+1}-d^{m}\right)^{2}-\frac{1}{16}\left(c^{m+1}-c^{m}\right)^{3} \\
& -\frac{3}{16}\left(c^{m+1}-c^{m}\right)^{2}\left(d^{m+1}-d^{m}\right)-\frac{3}{16}\left(c^{m+1}-c^{m}\right)\left(d^{m+1}-d^{m}\right)^{2}
\end{aligned}
$$

By the same arguments as used for (A.2), we get

$$
|I I| \leq C\left(\left(c^{m+1}-c^{m}\right)^{2}+\left(d^{m+1}-d^{m}\right)^{2}\right),
$$

where we used the fact that $\mathbf{c}^{m}, \mathbf{c}^{m+1}$ are bounded and omitted subscripts $i$ and $j$ for simplicity. From Eqs. (A.2) and (A.3), our assertion (A.1) follows.

Appendix B. Crank-Nicholson. Here, we present another scheme in which

$$
\hat{\phi}\left(\mathbf{c}^{n}, \mathbf{c}^{n+1}\right)=\frac{1}{2}\left(\mathbf{f}\left(\mathbf{c}^{n}\right)+\mathbf{f}\left(\mathbf{c}^{n+1}\right)\right) .
$$

This results in the more traditional (Crank-Nicholson) scheme:

$$
\begin{aligned}
\frac{\mathbf{c}_{i j}^{n+1}-\mathbf{c}_{i j}^{n}}{\Delta t} & =\Delta_{d} \boldsymbol{\mu}_{i j}^{n+\frac{1}{2}}, \\
\boldsymbol{\mu}_{i j}^{n+\frac{1}{2}} & =\frac{1}{2}\left(\mathbf{f}\left(\mathbf{c}^{n}\right)+\mathbf{f}\left(\mathbf{c}^{n+1}\right)\right)-\frac{1}{2} \boldsymbol{\Gamma}_{\epsilon} \Delta_{d}\left(\mathbf{c}_{i j}^{n}+\mathbf{c}_{i j}^{n+1}\right) .
\end{aligned}
$$

The nonlinear multigrid method given in section $\mathrm{C}$ also can be modified to solve this nonlinear system at the implicit time level. Moreover, at the linear level (i.e. f(c) is a linear function), this scheme is the same as that considered in (3.5) and (3.6). However, at the nonlinear level, we are unable to prove that the Crank-Nicholson system given above has a discrete energy function unless a second order time step constraint is imposed. This constraint is much stronger than that needed for stability and seems to be a shortcoming of the analysis as simulation results always seem to yield non-increasing discrete energies.

Appendix C. A nonlinear multigrid V-cycle algorithm.

Let us rewrite equations (3.5)-(3.6) as follows.

$$
\operatorname{NSO}\left(c^{n+1}, \mu^{n+\frac{1}{2}}, d^{n+1}, \nu^{n+\frac{1}{2}}\right)=\left(g_{1}^{n}, g_{2}^{n}, g_{3}^{n}, g_{4}^{n}\right),
$$


where the nonlinear system operator (NSO) is defined as

$$
\begin{aligned}
\operatorname{NSO}\left(c^{n+1}, \mu^{n+\frac{1}{2}}, d^{n+1}, \nu^{n+\frac{1}{2}}\right)= & \left(\frac{c_{i j}^{n+1}}{\Delta t}-\Delta_{h} \mu_{i j}^{n+\frac{1}{2}},\right. \\
\mu_{i j}^{n+\frac{1}{2}}-\hat{\phi}_{1}\left(c_{i j}^{n}, c_{i j}^{n+1}, d_{i j}^{n}, d_{i j}^{n+1}\right)+ & \epsilon^{2} \Delta_{h} c_{i j}^{n+1}+\frac{\epsilon^{2}}{2} \Delta_{h} d_{i j}^{n+1}, \\
& \frac{d_{i j}^{n+1}}{\Delta t}-\Delta_{h} \nu_{i j}^{n+\frac{1}{2}}, \\
\nu_{i j}^{n+\frac{1}{2}}-\hat{\phi}_{2}\left(c_{i j}^{n}, c_{i j}^{n+1}, d_{i j}^{n}, d_{i j}^{n+1}\right)+ & \left.\epsilon^{2} \Delta_{h} d_{i j}^{n+1}+\frac{\epsilon^{2}}{2} \Delta_{h} c_{i j}^{n+1}\right)
\end{aligned}
$$

and the source term is

$$
\left(g_{1}^{n}, g_{2}^{n}, g_{3}^{n}, g_{4}^{n}\right)=\left(\frac{c_{i j}^{n}}{\Delta t},-\epsilon^{2} \Delta_{h} c_{i j}^{n}-\frac{\epsilon^{2}}{2} \Delta_{h} d_{i j}^{n}, \frac{d_{i j}^{n}}{\Delta t},-\epsilon^{2} \Delta_{h} d_{i j}^{n}-\frac{\epsilon^{2}}{2} \Delta_{h} c_{i j}^{n}\right)
$$

In the following description of one FAS cycle, we assume a sequence of grids $\Omega_{k}$ $\left(\Omega_{k-1}\right.$ is coarser than $\Omega_{k}$ by factor 2$)$. Given the number $\eta$ of pre- and post- smoothing relaxation sweeps, an iteration step for the nonlinear multigrid method using the $\mathrm{V}$-cycle is formally written as follows:

\section{FAS multigrid cycle}

$$
\begin{gathered}
\left\{c_{k}^{m+1}, \mu_{k}^{m+\frac{1}{2}}, d_{k}^{m+1}, \nu_{k}^{m+\frac{1}{2}}\right\} \\
=F \operatorname{AScycle}\left(k, c_{k}^{m}, \mu_{k}^{m-\frac{1}{2}}, d_{k}^{m}, \nu_{k}^{m-\frac{1}{2}}, \mathbf{N S O}_{k}, g_{1 k}^{n}, g_{2 k}^{n}, g_{3 k}^{n}, g_{4 k}^{n}, \eta\right) .
\end{gathered}
$$

That is, $\left\{c_{k}^{m}, \mu_{k}^{m-\frac{1}{2}}, d_{k}^{m}, \nu_{k}^{m-\frac{1}{2}}\right\}$ and $\left\{c_{k}^{m+1}, \mu_{k}^{m+\frac{1}{2}}, d_{k}^{m+1}, \nu_{k}^{m+\frac{1}{2}}\right\}$ are the approximations of $\left\{c_{k}^{n+1}\left(x_{i}, y_{j}\right), \mu_{k}^{n+\frac{1}{2}}\left(x_{i}, y_{j}\right), d_{k}^{n+1}\left(x_{i}, y_{j}\right), \nu_{k}^{n+\frac{1}{2}}\left(x_{i}, y_{j}\right)\right\}$ before and after a FAScycle. Now, define the FAScycle.

\section{(1) Presmoothing}

$$
\begin{aligned}
& \left\{\bar{c}_{k}^{m}, \bar{\mu}_{k}^{m-\frac{1}{2}}, \bar{d}_{k}^{m}, \bar{\nu}_{k}^{m-\frac{1}{2}}\right\} \\
& =\operatorname{SMOOTH}{ }^{\eta}\left(c_{k}^{m}, \mu_{k}^{m-\frac{1}{2}}, d_{k}^{m}, \nu_{k}^{m-\frac{1}{2}}, \mathbf{N S O}_{k}, g_{1 k}^{n}, g_{2 k}^{n}, g_{3 k}^{n}, g_{4 k}^{n}\right),
\end{aligned}
$$

which means performing $\eta$ smoothing steps with initial approximation $c_{k}^{m}, \mu_{k}^{m-\frac{1}{2}}$, $d_{k}^{m}, \nu_{k}^{m-\frac{1}{2}}, g_{1 k}^{n}, g_{2 k}^{n}, g_{3 k}^{n}, g_{4 k}^{n}$, and the $S M O O T H$ relaxation operator to get the approximation $\left\{\bar{c}_{k}^{m}, \bar{\mu}_{k}^{m-\frac{1}{2}}, \bar{d}_{k}^{m}, \bar{\nu}_{k}^{m-\frac{1}{2}}\right\}$.

One SMOOTH relaxation operator step consists of solving the system (C.2)-(C.5) given below by a $4 \times 4$ matrix inversion for each $i j$ :

$$
\frac{\bar{c}_{i j}^{m}}{\Delta t}+\frac{4}{h^{2}} \bar{\mu}_{i j}^{m-\frac{1}{2}}=g_{1 i j}^{n}+\frac{\mu_{i+1, j}^{m-\frac{1}{2}}+\bar{\mu}_{i-1, j}^{m-\frac{1}{2}}+\mu_{i, j+1}^{m-\frac{1}{2}}+\bar{\mu}_{i, j-1}^{m-\frac{1}{2}}}{h^{2}},
$$




$$
\begin{gathered}
-\left(\frac{4 \epsilon^{2}}{h^{2}}+\frac{\partial \hat{\phi}_{1}}{\partial c_{i j}^{n+1}}\left(c_{i j}^{n}, c_{i j}^{m}, d_{i j}^{n}, d_{i j}^{m}\right)\right) \bar{c}_{i j}^{m}-\left(\frac{2 \epsilon^{2}}{h^{2}}+\frac{\partial \hat{\phi}_{1}}{\partial d_{i j}^{n+1}}\left(c_{i j}^{n}, c_{i j}^{m}, d_{i j}^{n}, d_{i j}^{m}\right)\right) \bar{d}_{i j}^{m} \\
+\bar{\mu}_{i j}^{m-\frac{1}{2}}=g_{2}^{n}+\frac{1}{2} \hat{\phi}_{1}\left(c_{i j}^{n}, c_{i j}^{m}, d_{i j}^{n}, d_{i j}^{m}\right)-\frac{\partial \hat{\phi}_{1}}{\partial c_{i j}^{n+1}}\left(c_{i j}^{n}, c_{i j}^{m}, d_{i j}^{n}, d_{i j}^{m}\right) c_{i j}^{m} \\
-\frac{\partial \hat{\phi}_{1}}{\partial d_{i j}^{n+1}}\left(c_{i j}^{n}, c_{i j}^{m}, d_{i j}^{n}, d_{i j}^{m}\right) d_{i j}^{m}-\frac{\epsilon^{2}}{h^{2}}\left(c_{i+1, j}^{m}+\bar{c}_{i-1, j}^{m}+c_{i, j+1}^{m}+\bar{c}_{i, j-1}^{m}\right) \\
-\frac{\epsilon^{2}}{2 h^{2}}\left(d_{i+1, j}^{m}+\bar{d}_{i-1, j}^{m}+d_{i, j+1}^{m}+\bar{d}_{i, j-1}^{m}\right) .
\end{gathered}
$$

Using similar procedures as above, we get Eqs. (C.4) and (C.5) from the second components of Eqs. (3.5) and (3.6), respectively:

$$
\begin{gathered}
\frac{\bar{d}_{i j}^{m}}{\Delta t}+\frac{4}{h^{2}} \bar{\nu}_{i j}^{m-\frac{1}{2}}=g_{3 i j}^{n}+\frac{\nu_{i+1, j}^{m-\frac{1}{2}}+\bar{\nu}_{i-1, j}^{m-\frac{1}{2}}+\nu_{i, j+1}^{m-\frac{1}{2}}+\bar{\nu}_{i, j-1}^{m-\frac{1}{2}}}{h^{2}} \\
-\left(\frac{2 \epsilon^{2}}{h^{2}}+\frac{\partial \hat{\phi}_{2}}{\partial c_{i j}^{n+1}}\left(c_{i j}^{n}, c_{i j}^{m}, d_{i j}^{n}, d_{i j}^{m}\right)\right) \bar{c}_{i j}^{m}-\left(\frac{4 \epsilon^{2}}{h^{2}}+\frac{\partial \hat{\phi}_{2}}{\partial d_{i j}^{n+1}}\left(c_{i j}^{n}, c_{i j}^{m}, d_{i j}^{n}, d_{i j}^{m}\right)\right) \bar{d}_{i j}^{m} \\
+\bar{\nu}_{i j}^{m-\frac{1}{2}}=g_{4 i j}^{n}+\hat{\phi}_{2}\left(c_{i j}^{n}, c_{i j}^{m}, d_{i j}^{n}, d_{i j}^{m}\right)-\frac{\partial \hat{\phi}_{2}}{\partial c_{i j}^{n+1}}\left(c_{i j}^{n}, c_{i j}^{m}, d_{i j}^{n}, d_{i j}^{m}\right) c_{i j}^{m} \\
-\frac{\partial \hat{\phi}_{2}}{\partial d_{i j}^{n+1}}\left(c_{i j}^{n}, c_{i j}^{m}, d_{i j}^{n}, d_{i j}^{m}\right) d_{i j}^{m}-\frac{\epsilon^{2}}{2 h^{2}}\left(c_{i+1, j}^{m}+\bar{c}_{i-1, j}^{m}+c_{i, j+1}^{m}+\bar{c}_{i, j-1}^{m}\right) \\
-\frac{\epsilon^{2}}{h^{2}}\left(d_{i+1, j}^{m}+\bar{d}_{i-1, j}^{m}+d_{i, j+1}^{m}+\bar{d}_{i, j-1}^{m}\right) .
\end{gathered}
$$

This a straightforward generalization of the smoother we used in [13] for binary system. See [13] for a derivation.

(2) Compute the defect

$$
\begin{aligned}
& \left(\overline{\operatorname{def}}_{1 k}^{m}, \overline{\operatorname{def}}_{2 k}^{m}, \overline{\operatorname{def}}_{3 k}^{m}, \overline{\operatorname{def}}_{4 k}^{m}\right) \\
& \quad=\left(g_{1_{k}}^{n}, g_{2 k}^{n}, g_{3 k}^{n}, g_{4 k}^{n}\right)-\mathbf{N S O}_{k}\left(\bar{c}_{k}^{m}, \bar{\mu}_{k}^{m-\frac{1}{2}}, \bar{d}_{k}^{m}, \bar{\nu}_{k}^{m-\frac{1}{2}}\right) .
\end{aligned}
$$

(3) Restrict the defect and $\left\{\bar{c}_{k}^{m}, \bar{\mu}_{k}^{m-\frac{1}{2}}, \bar{d}_{k}^{m}, \bar{\nu}_{k}^{m-\frac{1}{2}}\right\}$

$$
\begin{gathered}
\left(\overline{\operatorname{def}}_{1 k-1}^{m}, \overline{\operatorname{def}}_{2 k-1}^{m}, \overline{\operatorname{def}}_{3 k-1}^{m},{\overline{\operatorname{def}_{4 k-1}^{m}}}^{m}\right)=I_{k}^{k-1}\left(\overline{\operatorname{def}}_{1 k}^{m}, \overline{\operatorname{def}}_{2 k}^{m},{\overline{\operatorname{def}_{3 k}^{m}}}^{m}, \overline{\operatorname{def}}_{4 k}^{m}\right), \\
\left(\bar{c}_{k-1}^{m}, \bar{\mu}_{k-1}^{m-\frac{1}{2}}, \bar{d}_{k-1}^{m}, \bar{\nu}_{k-1}^{m-\frac{1}{2}}\right)=I_{k}^{k-1}\left(\bar{c}_{k}^{m}, \bar{\mu}_{k}^{m-\frac{1}{2}}, \bar{d}_{k}^{m}, \bar{\nu}_{k}^{m-\frac{1}{2}}\right) .
\end{gathered}
$$

(4) Compute the right-hand side

$$
\begin{aligned}
\left(g_{1 k-1}^{n}, g_{2 k-1}^{n}, g_{3 k-1}^{n}, g_{4 k-1}^{n}\right)= & \left(\overline{\operatorname{def}}_{1 k-1}^{m}, \overline{\operatorname{def}}_{2 k-1}^{m}, \overline{\operatorname{def}}_{3 k-1}^{m}, \overline{\operatorname{def}}_{4 k-1}^{m}\right) \\
& +\mathbf{N S O}_{k-1}\left(\bar{c}_{k-1}^{m}, \bar{\mu}_{k-1}^{m-\frac{1}{2}}, \bar{d}_{k-1}^{m}, \bar{\nu}_{k-1}^{m-\frac{1}{2}}\right) .
\end{aligned}
$$

(5) Compute an approximate solution $\left\{\hat{c}_{k-1}^{m}, \hat{\mu}_{k-1}^{m-\frac{1}{2}}, \hat{d}_{k-1}^{m}, \hat{\nu}_{k-1}^{m-\frac{1}{2}}\right\}$ of the coarse grid equation on $\Omega_{k-1}$, i.e.

$$
\mathbf{N S O}_{k-1}\left(c_{k-1}^{m}, \mu_{k-1}^{m-\frac{1}{2}}, d_{k-1}^{m}, \nu_{k-1}^{m-\frac{1}{2}}\right)=\left(g_{1_{k-1}^{n}}^{n}, g_{2 k-1}^{n}, g_{3 k-1}^{n}, g_{4 k-1}^{n}\right) \text {. }
$$


If $k=1$, we explicitly invert a $4 \times 4$ matrix to obtain the solution. If $k>1$, we solve (C.6) by performing a FAS $k$-grid cycle using $\left\{\bar{c}_{k-1}^{m}, \bar{\mu}_{k-1}^{m-\frac{1}{2}}, \bar{d}_{k-1}^{m}, \bar{\nu}_{k-1}^{m-\frac{1}{2}}\right\}$ as an initial approximation:

$$
\begin{array}{r}
\left\{\hat{c}_{k-1}^{m}, \hat{\mu}_{k-1}^{m-\frac{1}{2}}, \hat{d}_{k-1}^{m}, \hat{\nu}_{k-1}^{m-\frac{1}{2}}\right\}=\operatorname{FAScycle}\left(k-1, \bar{c}_{k-1}^{m}, \bar{\mu}_{k-1}^{m-\frac{1}{2}}, \bar{d}_{k-1}^{m},\right. \\
\left.\bar{\nu}_{k-1}^{m-\frac{1}{2}}, \mathbf{N S O}_{k-1}, g_{1 k-1}^{n}, g_{2 k-1}^{n}, g_{3 k-1}^{n}, g_{4 k-1}^{n} \eta\right) .
\end{array}
$$

(6) Compute the coarse grid correction (CGC)

$$
\begin{gathered}
\hat{v}_{1 k-1}^{m}=\hat{c}_{k-1}^{m}-\bar{c}_{k-1}^{m}, \quad \hat{v}_{2 k-1}^{m-\frac{1}{2}}=\hat{\mu}_{k-1}^{m-\frac{1}{2}}-\bar{\mu}_{k-1}^{m-\frac{1}{2}}, \\
\hat{v}_{3 k-1}^{m}=\hat{d}_{k-1}^{m}-\bar{d}_{k-1}^{m}, \quad \hat{v}_{4 k-1}^{m-\frac{1}{2}}=\hat{\nu}_{k-1}^{m-\frac{1}{2}}-\bar{\nu}_{k-1}^{m-\frac{1}{2}} .
\end{gathered}
$$

(7) Interpolate the correction

$$
\begin{array}{ll}
\hat{v}_{1 k}^{m}=I_{k-1}^{k} \hat{v}_{1 k-1}^{m}, & \hat{v}_{2 k}^{m-\frac{1}{2}}=I_{k-1}^{k} \hat{v}_{2 k-1}^{m-\frac{1}{2}}, \\
\hat{v}_{3 k}^{m}=I_{k-1}^{k} \hat{v}_{3 k-1}^{m}, & \hat{v}_{4 k}^{m-\frac{1}{2}}=I_{k-1}^{k} \hat{v}_{4 k-1}^{m-\frac{1}{2}} .
\end{array}
$$

(8) Compute the corrected approximation on $\Omega_{k}$

$$
\begin{aligned}
& c_{k}^{m, \text { after } C G C}=\bar{c}_{k}^{m}+\hat{v}_{1 k}^{m}, \quad \mu_{k}^{m-\frac{1}{2}, \text { after } C G C}=\bar{\mu}_{k}^{m-\frac{1}{2}}+\hat{v}_{2 k}^{m-\frac{1}{2}}, \\
& d_{k}^{m, \text { after } C G C}=\bar{d}_{k}^{m}+\hat{v}_{3 k}^{m}, \nu_{k}^{m-\frac{1}{2}}, \text { after } C G C=\bar{\nu}_{k}^{m-\frac{1}{2}}+\hat{v}_{4 k}^{m-\frac{1}{2}} .
\end{aligned}
$$

\section{(9) Postsmoothing}

$$
\begin{aligned}
& \left\{c_{k}^{m+1}, \mu_{k}^{m+\frac{1}{2}}, d_{k}^{m+1}, \nu_{k}^{m+\frac{1}{2}}\right\} \\
& =\operatorname{SMOOTH} H^{\eta}\left(c_{k}^{m, \text { after } C G C}, \mu_{k}^{m-\frac{1}{2}} \text {, after } C G C, d_{k}^{m} \text {, after } C G C,\right. \\
& \left.\nu_{k}^{m-\frac{1}{2}}, \text { after } C G C, \mathbf{N S O}_{k}, g_{1}^{n}, g_{2}^{n}, g_{3 k}^{n}, g_{4 k}^{n}\right) .
\end{aligned}
$$

This completes the description of a nonlinear FAScycle.

Appendix D. The first (J.S. Kim) and third (J. S. Lowengrub) authors acknowledge the support of the Department of Energy, Office of Basic Energy Sciences and the National Science Foundation. The authors are also grateful for the support of the Minnesota Supercomputer Institute, the Network \& Academic Computing Services (NACS) at UCI, and the hospitality of the Institute for Mathematics and its Applications.

\section{REFERENCES}

[1] J.W. Barrett and J.F. Blowey, An error bound for the finite element approximation of a model for phase separation of a multi-component alloy, IMA J. Numer. Anal., 16:257-287, 1996.

[2] J.W. Barrett and J.F. Blowey, An improved error bound for a finite element approximation of a model for phase separation of a multi-component alloy, IMA J. Numer. Anal., 19(1):147$168,1999$.

[3] J.W. Barrett and J.F. Blowey, Finite element approximation of a model for phase separation of a multi-component alloy with nonsmooth free energy and a concentration dependent mobility matrix, Math. Models Methods Appl. Sci., 9(5):627-663, 1999. 
[4] J.W. Barrett, J.F. Blowey and H. Garcke, On fully practical finite element approximations of degenerate Cahn-Hilliard systems, M2AN Math. Model. Numer. Anal., 35(4):713-748, 2001.

[5] J. W. Barrett and J. F. Blowey, Finite element approximation of an Allen-Cahn/Cahn-Hilliard system, IMA J. Numer. Anal., 22(1):11-71, 2002.

[6] V.E. Badalassi, H.D. Ceniceros and S. Banerjee, Computation of multiphase systems with phase field models, J. Comp. Phys., 190:371-397, 2003.

[7] J.F. Blowey, M.I.M. Copetti and C.M. Elliott, The numerical analysis of a model for phase separation of a multi-component alloy, IMA J. Numer. Anal., 16:111-139, 1996.

[8] M. Copetti, Numerical experiments of phase separation in ternary mixtures, Math. Comput. Simulation, 52(1):41-51, 2000.

[9] D.J. Eyre, Systems for Cahn-Hilliard equations, SIAM J. Appl. Math., 53(6):1686-1-712, 1993.

[10] H. Garcke, B. Nestler and B. Stoth, A multiphase field concept: numerical simulations of moving phase boundaries and multiple junctions, SIAM J. Appl. Math., 60(1):295-315, 2000.

[11] Junseok Kim, Modeling and simulation of multi-component, multi-phase fluid flows, Ph.D. thesis, School of Mathematics, University of Minnesota, 2002.

[12] Junseok Kim and John Lowengrub, Conservative Multigrid Methods for ternary Cahn-Hilliard Fluids, in preparation.

[13] Junseok Kim, Kyungkeun Kang, and John Lowengrub, Conservative multigrid methods for Cahn-Hilliard fluids, J. Comp. Phys., 193:511-543, 2004.

[14] John Lowengrub and Vittorio Cristini, in preparation.

[15] J.E. Morral and J.W. Cahn, Spinodal decomposition in ternary systems, Acta Metall., 19:1037$1045,1971$.

[16] D.A. Porter and K.E. Easterling, Phase Transformations in Metals and Alloys, van Nostrand, Reinhold, 1993.

[17] U. Trottenberg, C. Oosterlee, A. Schüller, MULTIGRID, Academic press, 2001. 